Revue européenne des sciences sociales

European Journal of Social Sciences

\title{
Georg Simmel. La dimension sociologique de la Wechselwirkung
}

Christian Papilloud

\section{(2) OpenEdition}

Édition électronique

URL : https://journals.openedition.org/ress/675

DOI : $10.4000 /$ ress. 675

ISSN : 1663-4446

Éditeur

Librairie Droz

Édition imprimée

Date de publication : 1 décembre 2000

Pagination : 103-129

ISBN : 2-600-00481-5

ISSN : 0048-8046

Référence électronique

Christian Papilloud, «Georg Simmel. La dimension sociologique de la Wechselwirkung », Revue européenne des sciences sociales [En ligne], XXXVIII-119 | 2000, mis en ligne le 15 décembre 2009, consulté le 21 septembre 2021. URL : http://journals.openedition.org/ress/675; DOI : https://doi.org/ $10.4000 /$ ress. 675 


\section{Christian PAPILLOUD}

\section{GEORG SIMMEL. LA DIMENSION SOCIOLOGIQUE DE LA WECHSELWIRKUNG ${ }^{1}$}

Le concept de Wechselwirkung est le principe organisateur de la pensée de Georg $\mathrm{Simmel}^{2}$. Repris de la physique par les tendances darwinistes et organicistes des premières sociologies ${ }^{3}$, la Wechselwirkung est utilisée par Simmel pour mettre en évidence la propriété spécifique du social: le relationnel. De là découle que, chez l'auteur, le sociologique ${ }^{4}$ signifie avant tout un relationnisme. Ceci lui permet d'y fonder son approche, qu'il est convenu aujourd'hui d'appeler le relativisme de Simmel ${ }^{5}$.

1 Cet article est tiré de notre thèse en cours, intitulée: Marcel Mauss, Georg Simmel. Eléments pour une approche sociologique de la relation humaine.

2 C'est notamment ce que Julien Freund aura, dans le contexte francophone de la réception des œuvres de Simmel, mis en évidence de façon décisive dans son article «De Max Weber à Georg Simmel» (J. Freund, 1991, pp. 14-21).

3 C'est ce que Tarde souligna déjà : «C'est la physique qui est le plus en faveur auprès des sociologues naissants, et, malgré, çà et là, des tendances marquées à regarder le groupe social comme une sorte d'organisme, le plus souvent il n'est question chez eux que de masse et de mouvement. 'Tout acte d'association est un acte de mouvement' dit Carey.» (G. Tarde, 1902, p. 57). A la façon générale de Tarde, nous dirions que 'darwinisme' et 'organicisme' ont certainement été des génériques d'affiliation d'une grande partie des premiers sociologues pour une raison cardinale: en comparant la société a un 'corps' humain doté d' 'organes', ils cherchaient à cerner des rythmes, des changements, bref, le mouvement propre qui fait 'société', sa 'basis', sa logique, son fonctionnement, sa structuration. Ainsi, pouvons nous trouver parmi ces courants:

1. dans le monde anglo-américain: Herbert Spencer (1820-1903), Walter Bagehot (1826-1877), Graham Sumner (1840-1910), Lester Ward (1847-1913), Franklin Giddings (1855-1931);

2. dans le monde austro-germanique: Ludwig Gumplowicz (1838-1909), Gustav Ratzenhofer (1842-1909), Otto Ammon (1842-1916);

3. en France: Clémence Royer (1830-1902), Paul Janet (1823-1899), Ernest Renan (1823-1892), Gustave Le Bon (1841-1931).

Rappelons enfin que l'idée de mouvement de vie et de processus va être exploité ensuite dans deux directions différentes. Ce sera d'une part l'apanage du courant de l'anthropologie philosophique, dont les représentants les plus célèbres sont venus de la philosophie allemande, de la biologie, de la psychiatrie, et de la psychanalyse. On y retrouve notamment H. Plesner, A. Gehlen, M. Scheler; on peut y affilier également V. von Weizsäcker, L. Szondi, etc. Ce sera d'autre part le personnalisme allemand avec M. Buber, F. Rosenzweig, E. Lévinas, etc. auquel se sont affiliés certains philosophes français comme par exemple G. Marcel. H. Bergson peut également être considéré proche de cette tendance, comme le suggère Fitzi (G. Fitzi, 1999).

4 Rappelons que le terme 'sociologique' chez Simmel ne change quasiment pas de sens tout au long de l'œuvre de l'auteur. Le sociologique est le caractère spécial de toute les formes possibles de la Wechselwirkung (G. Simmel, GSG 7, (1903) 1995, p. 167), ce que l'auteur répétera dans ses «Grundfragen» (G. Simmel, GSG 16, (1917) 1984, p. 27).

5 A ce propos, voir le remarquable article de Vandenberghe (F. Vandenberghe, 1999, pp. 132-144). 
C'est sur cet aspect, sociologique, de la Wechselwirkung que nous nous concentrerons dans cet article. Il peut être considéré comme un fil rouge des multiples significations que prend le concept dans l'œuvre de l'auteur. A partir de la dimension sociologique de la Wechselwirkung s'éloignent, se rapprochent ou se développent ses autres facettes, que l'on peut décrire comme ses dimensions psychosociale, métaphysique et méthodologique. Le fait de voir s'y manifester la plupart des concepts importants de Simmel (individu, société, sociation, échange, forme, contenu) est une preuve supplémentaire de la centralité de cette dimension sociologique pour l'étude de la Wechselwirkung de Simmel. Nous en mettrons en évidence le fondement que la Wechselwirkung partage avec les autres acceptions qu'en a Simmel, à savoir l'idée de processus ${ }^{6}$.

Nous manque toutefois ce que présuppose le développement de notre propos, à savoir la clarification du terme 'Wechselwirkung' lui-même. Il importe en effet de se demander d'abord d'où vient le mot Wechselwirkung, ce qu'il signifie en allemand, et comment Simmel se l'est approprié. Ce sera pour nous la possibilité par la suite d'en donner une interprétation pour la langue française. C'est par ce travail que nous commencerons.

\section{1. - 'WECHSELWIRKUNG'. ENTRE PHYSIQUE, PHILOSOPHIE ET LITTÉRATURE}

Le mot 'Wechselwirkung' est un composé de 'Wechsel' (changement, dans le sens de 'Tausch' (échange); désigne aussi un agir ou de l'argent qui sort d'un compte) $)^{7}$ et de 'Wirkung' (effet) ${ }^{8}$. Le mot 'wechsel' (de l'ancien Hochdeutsch 'wehsal') est probablement le plus ancien des deux.

Kant les réunit pour former le terme 'Wechselwirkung'. Il l'utilise pour exprimer ce que Newton entendait par 'mouvement des planètes'. Ce mouvement suppose une force d'attraction/répulsion entre les planètes propre à les maintenir à

6 L'idée de processus est fortement liée à la conception sociologique de la Wechselwirkung de Simmel. Voici comment il la présente dans «Über soziale Differenzierung»: «La résolution de l'âme de la société dans la somme des effets de changements de ses possesseurs est posée en direction de l'esprit de la vie moderne: résoudre le compact, lui-même identique, substantiel en fonction, force, mouvement et connaître dans tout Etre le processus de son devenir.» (G. Simmel, GSG 2, (1890) 1989, p. 130). Nous voyons ici que la Wechselwirkung apparaît à Simmel comme permettant de connaître ce processus qu'est la société. L'idée de processus revient constamment dans l'œuvre de l'auteur, comme ici, de façon exemplaire, dans sa «Soziologie»: «Aussi si vous 'vous attendez' - et certes pas seulement à une attaque extérieure, qui menace votre vie entière d'un coup - ainsi résumons-nous d'innombrables et ininterrompus processus, qui s'ouvrent à l'intérieur de cette image comme poussée et contre-poussée, danger et défense, répulsion et ré-association entre les éléments.» (G. Simmel, GSG 11, (1908) 1992, p. 556).

7 Nous nous référons ici à la définition qu'en donne le dictionnaire de l'ancien allemand de O. Shade (1882, entrée 'Wechsel').

$8 \mathrm{Au} \mathrm{VIII'}$ siècle, le mot 'Wirkung' a le sens de 'faire une empreinte', mais aussi de 'Werk', d' œuvre', d' 'être capable', d' 'être responsable', de 'travail'. Il devient au XVIII' siècle, 'faire impression', 'avoir de l'influence'. Voir notamment le dictionnaire étymologique de W. Pfeifer (1989, entrée 'Wirkung').

$9 \quad$ Isaac Newton, Optique, Paris, Bourgois, (1787) 1989. 
distance les unes des autres. Kant développe sa conception de la Wechselwirkung dans la deuxième partie de la Kritik der reinen Vernunft ${ }^{10}$. Il y montre que le mouvement décrit par Newton n'appartient pas seulement à l'ordre de la matière; il vaut également pour l'espèce humaine. Le libellé ajouté au paragraphe concernant la Wechselwirkung lors de la seconde édition de la Kritik ${ }^{11}$ laisse en outre supposer que Kant utilise cette notion dans le sens d'un continuum reliant l'espace et le temps.

A partir de Kant, le lexique de la Wechselwirkung s'enrichit. Nous voyons apparaître le verbe 'wechselwirken' dans un poème lyrique de Friedrich Rückert (1788-1866) Der Bau der Welt'. Nous trouvons mentions du terme 'Wechselwirksamkeit' dans les Grundriss des Eigenthümlichen der Wissenschaftslehre $(1795)^{13}$ de Johann Gottlieb Fichte. Le champ sémantique de la Wechselwirkung s'accroît encore dès le début du XIXème siècle avec Goethe ${ }^{14}$, Schlegel ${ }^{15}$, Hegel ${ }^{16}$,

10 Immanuel Kant, Kritik der reinen Vernunft, Frankfurt am Main, Suhrkamp, W. Weischedel (eds), (1787) 1995. La loi de Wechselwirkung apparaît dans le cadre de l'analytique transcendantale, au sous-point consacré à l'analytique des concepts qui, dans la première version de la Kritik (1781), s'intitule: «Grundsatz der Gemeinschaft». Le titre sera précisé dans la seconde version de l'ouvrage (1787) de la façon suivante: «Grundsatz des Zugleichseins, nach dem Gesetze der Wechselwirkung, oder Gemeinschaft» (I. Kant, (1787) 1995, A 211, p. 242). Il faut peut-être encore préciser que le concept de Wechselwirkung apparaît chez Kant dès 1755 dans Allgemeine Naturgeschichte und Theorie des Himmels (I. Kant, (1755) 1902-1923, I, pp. 266-364). Mais il reste circonscrit dans le discours de Kant au contexte de la nature et de l'astronomie. Il ne se développe dans le sens qu'exposera la Kritik der reinen Vernunft qu'aux environs de 1765-66, dès Nachricht von der Einrichtung seiner Vorlesungen in dem Winterhalbenjahre von 1765-1766 (I. Kant, (1765-66) 1902-1923, II, pp. 313 ssq.) et Träume eines Geistersehers (I. Kant, (1766) 1902-1923, II, pp. 335 ssq.).

11 «Alle Substanzen, sofern sie im Raume als zugleich wahrgenommen werden können, sind in durchgängiger Wechselwirkung.» (I. Kant, (1787) 1995, A 211, p. 242).

12 Voir à ce propos: P. Christian, 1978, p. 110.

13 En particulier Johann Gottlieb Fichte, (1795) 1845-1846, I, p. 406. Le mot 'Wechselwirksamkeit' apparaît également dans Grundlage des Naturrechts (J. G. Fichte, (1796) 1845-1846, III, p. 34). Fichte l'utilise dans les deux cas pour expliquer la manière dont le Je se constitue dans une relation à un Non-Je, relation de laquelle naîtrait le sujet comme sujet moral. Il est encore à souligner que Fichte utilise le mot Wechselwirkung dans son œuvre dès les environ de 1792, et en deux sens différents. La Wechselwirkung se présente comme le principe méthodologique d'unité de la perception (par exemple, J. G. Fichte, (1792) 1845-1846, II, p. 4), aussi bien que comme celui de l'unité du Je dans son rapport constitutif au non-Je (par exemple, J. G. Fichte, (1795) 1845-1846, I, p. 369).

14 Goethe emploie 'Wechselwirkung' dans un sens général, pour décrire un état d'ouverture originaire de l'art et de la science au monde. La Wechselwirkung est un principe de relation d'emblée universel, qui va à l'encontre de toute forme de fermeture, ainsi que Goethe l'expose dans son aphorisme «Es gibt keine patriotische Kunst»: «Es gibt keine patriotische Kunst und keine patriotische Wissenschaft. Beide gehören wie alles hohe Gute der ganzen Welt an und können nur durch allgemeine freie Wechselwirkung aller zugleich Lebenden in steter Rücksicht auf das, was uns vom Vergangenen übrig und bekannt ist, gefördert werden.» (J. W. von Goethe, (1832) 1919, vol. 42, T. I, pp. 195-196).

15 Cf. August Wilhelm von Schlegel, Über Literatur, Kunst und Geist des Zeitalters : eine Auswahl aus den kritischen Schriften, Stuttgart, F. Finke (eds), Reclam, (1802) 1994.

16 Hegel présente la Wechselwirkung conformément à sa conception de la logique, comme lieu d'un proto-rapport de causalité ouvrant sur l'infini, comme ceci apparaît de façon exemplaire dans le premier volume de son Enzyklopädie der philosophischen Wissenschaften (1830): «In der 
Schleiermacher ${ }^{17}$ et Dilthey ${ }^{18}$. Ils font entrer définitivement la Wechselwirkung dans le domaine de l'humain.

L'usage kantien du mot 'Wechselwirkung' renvoyait à une loi du mouvement commune à la nature et aux hommes ${ }^{19}$. Depuis le début du XIX ${ }^{\mathrm{e}}$ siècle, sa signification se spécifie en rapport au monde humain. La Wechselwirkung prend place

Wechselwirkung, obgleich die Kausalität noch nicht in ihrer wahrhaften Bestimmung gesetzt ist, ist der Progreß von Ursachen und Wirkungen ins Unendliche als Progreß auf wahrhafte Weise aufgehoben, indem das geradlinige Hinausgehen von Ursachen zu Wirkungen und von Wirkungen zu Ursachen in sich um- und zurückgebogen ist.» (G. W. F. Hegel, (1830) 1998, p. 487; voir également le $\S 155$ qui suit, et qui est spécifiquement consacré à la Wechselwirkung). La Wechselwirkung sera par la suite identifiée à la causalité, comme Hegel l'indique dans le second volume de son Wissenschaft der Logik (G. W. F. Hegel, (1831) 1998, p. 350).

17 Schleiermacher donne une connotation dynamique et, originalité remarquable, sociale à la Wechselwirkung. Elle est ce caractère sociable de toute activité humaine, qui en contient toutes les formes et tous les buts, et qui fait apparaître l'être véritable de la société: «Die Wechselwirkung ist sonach in sich selbst zurückgehend und vollendet; in den Begriff derselben ist sowohl die Form als der Zweck der geselligen Tätigkeit enthalten, und sie macht das ganze Wesen der Gesellschaft aus.» (F. Schleiermacher, (1927), Vol. 2, p. 10). La Wechselwirkung est à la base de la loi quantitative de la sociabilité qui s'énonce de la manière suivante: «[...] deine gesellige Tätigkeit, soll sich immer innerhalb der Schranken halten, in denen allein eine bestimmte Gesellschaft als ein Ganzes bestehen kann.» (F. Schleiermacher, (1927), vol. 2, p. 11).

18 Dilthey introduit le terme de Wechselwirkung à partir d'une réflexion sur le contexte d'effets (Wirkungszusammenhang) du monde de l'esprit, sur lequel il insiste plus particulièrement à l'occasion de son écrit Der Aufbau der Geschichtlichen Welt in den Geisteswissenschaften (W. Dilthey, vol. VII, (1883) 1961, ch. 4). Le monde des effets est directement distingué du monde de la causalité, comme le monde de l'esprit l'est du monde de la nature: «Dieser Wirkungszusammenhang unterscheidet sich von dem Kausalzusammenhang der Natur dadurch, dass er nach der Struktur des Seelenlebens Werte erzeugt und Zwecke realisiert.» (W. Dilthey, vol. VII, (1883) 1961, p. 153). Le monde de la Wechselwirkung comme Wirkungszusammenhang est mis en relation avec la vie en générale et la communauté humaine-sociale en particulier: «[...] Die Lebenseinheit ist ein Wirkungszusammenhang, der vor dem der Natur voraus hat, dass er erlebt wird, dessen wirkende Teile aber nicht nach ihrer Intensität gemessen, sondern nur abgeschätzt werden können, dessen Individualität vom Gemeinschaftlich-Menschlichen nicht loslösbar ist, so dass Menschheit nur ein unbestimmter Typus ist.» (W. Dilthey, vol. VII, (1883) 1961, p. 159). Toutefois, le monde des effets, et partant de la Wechselwirkung, et rapporté largement au monde de l'individu saisit moins comme unité psycho-physique que comme unité de vie: «Wie stark auch jeder psychische Vorgang, der einem solchen Ganzen angehört, durch die Intention des Wirkungszusammenhangs bestimmt sein mag, immer ist dieser Vorgang nicht von dieser Intention ausschliesslich bestimmt. Das Individuum, in dem er sich vollzieht, greift als Lebenseinheit in den Wirkungszusammenhang ein; in seiner Äusserung ist es als Ganzes wirksam.» (W. Dilthey, vol. VII, (1883) 1961, pp. 159-160).

19 On peut suivre en ce sens Christian, lorsqu'il affirme: «Bereits bei Kant werden ja aus 'Bewegungen vermittels ihrer Wechselwirkung Kreisbewegungen', die 'in eine gemeinschaftliche Richtung' ausschlagen; und dass sich alle Substanzen, sofern sie zugleich sind, in Wechselwirkung unter einander befinden, heisst, dass sie 'in durchgängiger Gemeinschaft stehen'. Zwar geht das Wort 'Gemeinschaft' bzw. 'gemeinschaftlich' ursprünglich auf 'gemein' zurück, dessen Wurzel die Bedeutung von 'wechseln, tauschen' und 'mehreren im Wechsel zukommend' enthält, es wäre aber denkbar, dass Kant hier bereits der Bedeutungsverschiebung des Wortes 'Gemeinschaft' im Sinne von 'Zusammengehörigkeit' und 'Verbundenheit' Rechnung trägt. Für Goethe jedenfalls stellen sich die in ewiger Wechselwirkung begriffenen Momente als ein 'belebtes Ganze' dar und für Schlegel offenbart sich uns die unendliche Wechselwirkung im 'Organismus'.» (P. Christian, 1978, pp. 114-115). 
dans l'entre-deux des hommes qu'elle remplit et limite ${ }^{20}$. Dans l'acception de Goethe, Schlegel et aussi de Hegel, elle induit la perspective d'un infini ${ }^{21}$ qui lie les hommes, connotation qui lui reste par la suite fortement attachée. Avec Schleiermacher et Dilthey, la Wechselwirkung devient le principe de l'agir social. Comment Simmel reçoit-il ce terme, et comment l'utilise-t-il dans ses propres travaux?

\section{2. - LA WECHSELWIRKUNG DE SIMMEL. HÉRITAGES ET FORMULATIONS}

Simmel reprend le mot 'Wechselwirkung' pour l'articuler à son travail qu'il considère être une philosophie avant tout ${ }^{22}$. Il n'est dès lors pas étonnant que l'usage du terme renvoie chez lui en priorité à Kant. La Wechselwirkung de Simmel évoque un mouvement d'attraction/répulsion dans le monde humain. Elle intègre la perspective d'un infini. Mais elle n'est pas directement le principe d'un agir social. Elle est d'abord 'matérielle', en ce sens qu'elle contient l'ensemble des contenus de la vie ${ }^{23}$. Qualifier la Wechselwirkung de matérielle, c'est revenir au concept de matière de Kant sans en reprendre le contenu. C'est en outre se tenir

20 Nous suivons en ce sens la remarque de Christian: «Seit dem Aufkommen des Wortes 'Wechselwirkung' in der Mitte des 18. Jahrhunderts verbindet sich mit diesem Wort die Bedeutung einer ineinandergreifenden, sich wechselseitig ergänzenden, aber auch begründenden oder bedingenden und begrenzenden Wirkung meist zwischen zweien.» (P. Christian, 1978, p. 114).

21 C'est ce que Christian met bien en évidence ici: «Auffallend ist die nähere Kennzeichnung der Wechselwirkung - etwa bei Goethe und Schlegel - durch Adjektive wie 'ewig' und 'unendlich'.» (P. Christian, 1978, p. 114). Cette connotation est par la suite encore plus accentuée par d'autres auteurs allemands. Chez Herder et Schlegel on retrouve la 'durchgängige Wechselwirkung', chez Raupach la 'stete Wechselwirkung', chez Freytag la 'unaufhörliche Wechselwirkung', chez von Bismark la 'ununterbrochene Wechselwirkung', chez Gützow la 'ewige Wechselwirkung', chez W. von Humboldt la 'lebendige Wechselwirkung', et chez A. von Humboldt la 'tätige Wechselwirkung'. Tout ces adjectif expriment un développement infini de la Wechselwirkung.

22 N'oublions pas que Simmel se considère comme philosophe plus que comme sociologue, ce qu'il dit à Jellinek en ces termes: «Que je ne suis pas philosophe en vérité, mais sociologue, est une absurdité. En réalité, je ne fais de la sociologie que comme emploi accessoire.» (Lettre à Jellinek du 20.03.1908, Archives Georg Simmel, Université de Bielefeld).

23 Le mot 'matériel' se lie chez Simmel au mot 'matière', évoqué le plus souvent en relation avec le concept de forme. De claires mentions de ce rapprochement se trouvent fréquemment évoquées dans les textes mineurs de Simmel, comme par exemple «Soziologische Aesthetik» (G. Simmel, GSG 5, (1896) 1992, pp. 197 et ssq.), «Die Alpen» (G. Simmel, GSG 14, (1908) 1996, p. 298), «Die Ruine. Ein ästhetischer Versuch» (G. Simmel, GSG 14, (1907) 1996, pp. 287-290). Les textes les plus importants sur ce point n'en restent pas moins: «Das Problem der Soziologie» (GSG 5, (1894) 1992; GSG 11, (1908) 1992, pp. 13-42), le second chapitre de la Philosophie des Geldes, à savoir «Der Substanzwert des Geldes» (GSG 6, (1900) 1996, pp. 139173), et l'essai «Aesthetik der Schwere» (GSG 7, (1901) 1995). Et pour autant qu'il faille risquer une citation, nous choisirions la suivante: «Je décris maintenant tout ce qui dans les individus existe à ce point comme le lieu direct et concret de toutes les vérités historiques comme pulsion, intérêts, buts, négation, état psychique et mouvement, dont découle ou qui induit l'effet sur les autres et le ressenti de leurs effets - ceci je le décris comme le contenu, en même temps la matière de la sociation.» (G. Simmel, GSG 11, (1908) 1992, p. 18). 
à distance de Schleiermacher et Dilthey, pour qui la Wechselwirkung est d'emblée le principe de l'action sociale. Sa thèse de doctorat ${ }^{24}$, bien que le mot même de Wechselwirkung n'y apparaisse pas ${ }^{25}$, l'annonce à travers une critique de la monadologie physique kantienne. Exposons-en brièvement le propos.

Pour Simmel, Kant pense toujours selon la représentation leibnizienne du monde. Il le remarque en lisant ses réflexions sur les travaux de Newton et d'Euler, où Kant s'intéresse à l'idée de monade (ce que Simmel nomme 'Ding an sich', c'est-à-dire 'chose en soi'; G. Simmel, GSG 1, 1881, p. 32) ${ }^{26}$. Pour le philosophe de Königsberg, les monades peuvent être rassemblées en éléments formant un tout. Elles prennent place dans un espace délimité qu'elles peuvent remplir selon l'effet ('Wirkung', G. Simmel, GSG 1, 1881, p. 9) qu'elles ont sur lui. Mais Simmel objecte que: «[...] la représentation selon laquelle la monade remplit l'espace à travers sa capacité de faire effet ne peut être complétée, parce que cet effet ne

24 G. Simmel, 1881 (25 Février), Das Wesen der Materie nach Kant's Physischer Monadologie, Berlin, Druck der Norddeutschen Buchdruckerei (publiée récemment dans les œuvres complètes, cf. GSG 1, 2000, pp. 9-43). Rappelons que ce ne fut pas, à l'origine, la thèse de doctorat de Simmel, qui s'intitulait «Psychologisch-ethnographische Studien über die Anfänge der Musik» (publié dans la Zeitschrift für Völkerpsychologie und Sprachwissenschaft en 1882; disponible dans le GSG 1, 2000, pp. 45-88). L'écrit sur Kant fut proposé comme thèse de doctorat à la place de ce dernier texte sur les conseils de Helmoltz, l'un des deux jurés de thèse de Simmel avec l'historien H. Zeller. Helmoltz et Zeller avaient jugé que la critique de Simmel contre Darwin sur l'origine du langage ne pouvait pas être acceptée. Simmel tentait de montrer contre Darwin que ce n'est pas le chant qui aurait permis le développement de la langue, mais qu'au contraire le chant supposait la possession de la parole: «Rien ne me semble plus probable que le chant, à son origine, fut une croissance de la parole à travers l'affect, à partir du rythme et de la modulation.» (G. Simmel, GSG 1, (1882) 2000, p. 48).

25 La première mention du terme en tant que tel par Simmel se trouve évoquée dans son texte de 1888, «Bemerkungen zu socialethischen Problemen», Vierteljahresschrift für wissenschaftliche Philosophie, $\mathrm{n}^{\circ} 12$ : 40. Ce texte quasiment inconnu, récemment disponible dans le GSG 1 (2000, pp. 221-232), fait figure rétrospectivement de plan de travail théorique que Simmel semble s'être donné dans sa jeunesse. Il y trace les lignes générales de ses principales problématiques, et annonce déjà ses plus grands travaux.

26 Rappelons que, pour Simmel, le 'Ding an sich' de Kant est quelque chose que la connaissance n'épuise pas, à moins de postuler une connaissance pure, qui n'existe pas. Simmel fait également la démonstration inverse, et reprend le 'Ding an sich' dans le sens kantien du 'Je'. Or, dit-il, le 'Je' est ce qui se dérobe in fine à la connaissance; la connaissance est donc imparfaite. On retrouve chez Simmel les traces de ce développement dans deux textes principaux: «Was ist uns Kant?» (GSG 5, (1896) 1992) et «Venedig» (GSG 8, (1907) 1993, p. 259 notamment). Evidemment, tout le problème de cette argumentation est qu'elle ne repose sur rien de formel ou de très structuré. La question suivante reste en suspens : pourquoi ne peut-on connaître le 'Je' complètement? L'un quelconque des durkheimiens, contemporain de Simmel, y aurait sans doute vu un laisser-aller théorique indigne du sociologue. Mais c'est peut-être manquer le propos de l'auteur que de se positionner ainsi. Simmel déplace en fait la question philosophique du 'Je' sur un terrain autre que celui de l'épistémologie classique (à savoir la théorie de la connaissance kantienne), dans la vie quotidienne, dans ses variations innombrables telles qu'elles se manifestent dans les relations sociales que les gens entretiennent entre eux. Pour la sociologie, dit-il, il n'y a pas de 'Je' qui soit entièrement connaissable, même s'il est le motif de l'individuel ou sa valeur. Tout un ensemble de sous-problématiques sont par là mises au devant de la scène, dans une nouvelle formulation de la question du 'Je': la vérité, la réalité, l'altérité, la légitimité du social, la relevance épistémologique de la sociologie, la considération de son statut scientifique, etc. Il faudra par la suite être attentif à ce genre de formulation, qui soulève des lièvres plus qu'elle ne cherche une réponse idéale. 
peut que retrouver des monades qui se présentent à lui sans espace.» (G. Simmel, GSG 1, 1881, p. 9). La structure de l'espace est bien, pour Simmel comme pour Kant, la résultante d'une force d'attraction/répulsion. Mais pour Simmel, cette force n'appartient pas aux monades, au contraire de ce qu'affirme Kant, chez qui «[...] la force, laquelle ne devrait pourtant être qu'une relation entre monades, est formellement hypostasiée à une substance élargie.» (G. Simmel, GSG 1, 1881, p. 13). Le mouvement, telle que le pense Simmel, n'est donc pas lié à la matière de la monade. C'est un processus de régulation des monades. Il se situe entre elles et procède selon l'aller et le retour. Tel est le noyau du matériel, du mouvement, et partant de la Wechselwirkung chez Simmel. Tel est le point où il rencontre la tradition l'ayant précédé, tout en y marquant sa différence.

Nous voyons ainsi que la Wechselwirkung, en dépit de son clair rapport avec le domaine de l'homme, n'est pas d'emblée un 'lien social'. Ce n'est jusqu'ici qu'un processus, dont les caractères (psychiques, psychologiques, moraux, etc.) doivent s'affirmer ou mieux, sont affirmés selon son mouvement même. Ce mouvement fait de l'effet, change, fait changer, suppose l'échange. C'est par ces termes que l'on peut rendre en français une signification approximative du mot allemand 'Wechselwirkung'. Et s'il fallait en donner une expression synthétique, nous choisirions 'effet(s) de changement(s)'. Cette formule nous paraît suffisamment souple pour s'adapter à ce qu'évoque l'allemand 'Wechselwirkung', et qui reste intraduisible en français. La structure minimale du terme peut alors être décrite ainsi: il y a un mouvement d'attraction et de répulsion qui fait effet et qui change, dont sortent des effets de changements échangés. La Wechselwirkung n'est donc pas d'emblée la 'réciprocité' 27 , 'l'action réciproque' ou encore 'l'interaction' que veulent y voir la plupart des traductions des œuvres de Simmel que nous avons pu consulter ${ }^{28}$. Ces notions empêchent en outre de considérer les

27 C'est en ce sens restreint qu'Aron par exemple comprend la Wechselwirkung chez Simmel:

«L'essence de la vie sociale implique un minimum de réciprocité (...), résonnance de l'homme à l'homme, élargissement du moi au-delà de ses limites. La conscience individuelle est primitivement 'hors d'elle-même'.» (R. Aron, (1935) 1981, p. 27). Mais c'est oublier ici que la réciprocité ne recouvre pas la Wechselwirkung, qui exprime à la différence de la réciprocité un mouvement sur lequel les individus n'ont pas complètement prise. Il y a toutefois, reconnaissons-le, une affinité certaine entre les deux termes du point de vue du mouvement d'aller et de retour qui caractérise la Wechselwirkung, et que le mot réciprocité (re-pro-cos, qui va en arrière et en avant) évoque. Ce n'est peut-être d'ailleurs pas pour rien que Simmel écrira son «Panta rhei » (1917) qui évoque Héraclite, et sa fameuse dialectique des contrariétés. Mais encore faut-il se garder de réduire la Wechselwirkung à la réciprocité, comme l'a fait encore Jankélévitch, au nom de la seule vertu évocatrice du terme (V. Jankélévitch, 1925, pp. 213-257 et pp. 373-386).

28 Non seulement les traductions ou références en français à Simmel traduisent la Wechselwirkung ainsi, mais également les traductions anglaises, et sans doute d'autres encore. Donnons-en quelques exemples parmi la littérature contemporaine: «L'objectivation des contenus de notre vie ne dépend donc pas de notre mortalité mais de l'objectivité qui est produit par l'interaction sociale.» (A. Hahn, 1988, p. 4); «L'action réciproque (Wechselwirkung) est au centre de la démarche concrète de Simmel. Elle exprime justement l'incessant jeu de variations dans les interactions humaines. (...) L'idée de l'action réciproque exige tout d'abord que l'on prenne en compte l'idée que Simmel se faisait de l'action. (...) Le concept d'action implique à son avis que l'on organise les moyens en vue d'atteindre une fin déterminée et particulière qui donne cohérence à l'entreprise singulière à laquelle on se livre.» (J. Freund, 1992, p. 71-72); «He advanced, instead, the conception that society consists of a web of patterned interactions, and that it is the task of sociology to study the forms of these interactions as they occur and reoccur in diverse historical 
emplois particuliers que Simmel en fait, puisque chez lui elles ne sont pas équivalentes entre elles ${ }^{29}$. Simmel aurait pu le préciser lui-même, pour éviter les mésinterprétations toujours possibles de son œuvre; mais il ne le fait pas. Il n'y a pas de recours à de quelconques sources ou auteurs sur ce point, pas plus qu'à des études philologiques $^{30}$. Pourquoi?

Nous pouvons y voir un des effets du style artiste de Simmel, le mot ne référant à rien d'ironique ou de dilettante ${ }^{31}$. Il renverrait plutôt à l' « Ausbildung» goethéenne que Die Leiden des jungen Werthers dépeint ${ }^{32}$. L'écrit se veut œuvre d'art, et cela presque dès le début de la production intellectuelle de Simmel. Les réfé-

periods and cultural settings.» (L. Coser, 1977, p. 193); «The a prioris of social interaction appear, not in the mind of the sociologist, but in the minds of actors he or she studies. In order for persons to interact at all, they must form images of one another. This means, for example, viewing one another in ways that disort the other's concrete individuality, adding traits here and substracting them there. We generalize about one another with respect to the type of person the other represents, with respect to idealized notions of the other, and with respect to the qualities of others as members of particular social groups. We conceive of the other as simultaneously functioning within a social role and functioning as an autonomous being outside such a role." (D. Levine, 1988, p. 12); «Simmel adopted Kant's method of first considering the existence of some study or some object and then asking what are the conditions that make it possible. (...) And, he borrowed from Kant the fundamental concept of reciprocal interaction or community. (...) Simmel adopts this notion and maintains that it is this condition of mutual interaction that makes society possible.» (Ch. Adair-Toteff, 1994, p. 3). Ces quelques exemples, qui pourraient être allongés facilement, montrent suffisamment que la question de la Wechselwirkung n'est pas posée. Au fond, la plupart des commentateurs de Simmel, et ceci indépendamment de la qualité des travaux qu'ils réalisent, renouent avec le syndrome de la découverte de l'Amérique: on découvre l'autre à travers l'imposition (et non la discussion) de ses propres catégories de pensée. Il n'est dès lors pas étonnant que nous retrouvions du Simmel dans une bonne partie de la sociologie moderne, bien que peut-être Simmel lui-même ne s'y (re)trouverait nulle part.

29 C'est ainsi par exemple que la Wechselwirkung traverse les écrits de l'auteur, alors que la réciprocité ('Gegenseitigkeit') n'est évoquée qu'à quelques reprises (voir notamment G. Simmel, GSG 6, (1900) 1996, pp. 55-93, pp. 585 et ssq.), et que nous ne trouvons pas mention à notre connaissance du concept d'interaction. En outre, il n'est pas difficile de constater que la Wechselwirkung est utilisée comme concept abstrait, alors que la réciprocité désigne chaque fois une situation de co-présence concrète. En ce sens, la réciprocité est bien une forme de Wechselwirkung, mais l'inverse est faux ou ne peut-être dit.

30 Pourtant Simmel connaît plusieurs langues: le français, l'anglais, l'italien, le latin, le grec ancien. Sa formation de philosophe, la tradition allemande des études de philologie aurait pu le prédisposer à entreprendre ce genre de recherche. Mais il n'en est rien.

31 Par style artiste, il faut surtout comprendre, comme nous y encourage Frisby, que contrairement au travaux de Weber et de Durkheim, Simmel ne s'est pas attaché à rassembler la multitude des faits. «It is all the more remarkable then that after his death attempts were made to order his sociological writings under the rubric of 'systematic' sociology, despite his persistent and explicit aversion to all forms of system.» (D. Frisby, 1984, p. 16). Simmel peut également apparaître comme le microscopiste de la société, selon le mot de Nisbet, ou comme son Freud, selon l'expression de Hughes (cf. R. A. Nisbet, 1966, pp. 97-98). Jusqu'à quel point est-il un flâneur (au sens de W. Benjamin; voir D. Frisby, 1981, pp. 68-101) ou un bricoleur (D. Weinstein, A. Weinstein, 1993, pp. 53-71) en sociologie, c'est difficile à déterminer. Nous aurons tendance de notre côté à penser que Simmel possède un projet scientifique cohérent, mais dont il cherche une systématique qui ne réifie pas la complexité de l'objet d'étude: les relations sociales et au-delà, leur devenir.

32 (J.W. Goethe, 1786). Il faut toutefois préciser d'emblée que Simmel reste à distance de l'image idéale et harmonieuse qu'évoque la vie de Goethe, comme il l'exprime dans son journal: «Non sens que la vie doit être faite en direction d'une œuvre d'art.» (G. Simmel, 1919/20, pp. 136). 
rences à quelques auteurs ou conceptions théoriques sont par conséquent rarement explicitées, et lorsque c'est le cas, le texte n'est pas toujours respecté. Ce n'est pas que Simmel cite au hasard de la mémoire, comme Durkheim l'avait cru ${ }^{33}$ : il retravaille ses citations comme pour les 'adapter' à son style d'écriture. Il ne fait presque aucune notes en bas de page, si ce n'est pour préciser sa propre pensée, et ne propose pas de bibliographie. Son écriture ne se soucie pas de la forme scientifique. Simmel privilégie la suggestion et la fiction, le 'als ob'34 ('comme si') amené par des remarques subtiles et profondes, comme l'observait déjà Bouglé 35 . La sociologie, qui lui apparaît comme une «[...] histoire de la société et de tous ses contenus, c'est-à-dire au sens d'une explication de tout devenir au moyen des forces et configurations sociales, est si peu une science particulière, comme l' 'induction' par exemple.» (G. Simmel, GSG 5, (1894) 1992, p. 53) ${ }^{36}$. C'est une méthode, un principe heuristique qui peut fructifier de façon infinie le domaine de la connaissance (G. Simmel, GSG 5, (1894) 1992, p. 53). Telle est pourrait-on dire à un niveau très général le rôle de la Wechselwirkung dans l'œuvre de Simmel. Elle évoque, elle donne à penser par image, elle guide l'esprit vers le mouvant et le mouvement. C'est peut-être la raison pour laquelle son discours s'adapte si bien à sa perspective théorique fondamentale: la relation humaine entendue comme processus de vie, enraciné dans le mouvement des Wechselwirkungen que les individus portent les uns vers les autres comme autant d'effets qui changent et les font é-changer. C'est probablement pour cela que lorsque nous lisons Simmel, l'eau, la fluidité, le vent, la musique nous viennent à l'esprit.

Du style d'écriture au style de recherche que propose Simmel pour l'étude de la société ou plus précisément de la sociation ('Vergesellschaftung') ${ }^{37}$, le chemin est tout tracé. Il est l'œuvre d'un homme étriqué dans le seul regard du philosophe ou du sociologue, qui oscille en permanence entre philosophie et sociologie. Ce balancement ininterrompu d'une orientation à l'autre, doublé du devoir de science stylisée auquel Simmel tient, mène à l'approfondissement du monde de la Wechselwirkung. Plus que de recevoir une définition, la Wechselwirkung est mise en quête de sa définition. Comment se présente-t-elle au niveau du registre sociologique? Telle est le point que nous développons dans ce qui suit.

A ce propos, voir C. Papilloud, 2000, pp. 114-132.

34 Simmel s'en sert très régulièrement dans toute son œuvre. On peut peut-être en voir la manifestation la plus fréquente dans la troisième partie du premier chapitre de Philosophie des Geldes (G. Simmel, GSG 6, (1900) 1996, pp. 93-139).

35 A ce sujet, voir notamment l'article de Bouglé 'La sociologie de Georg Simmel' in Ch. Andler, V. Basch, J. Benrubi, C. Bouglé, V. Delbos, G. Dwelshauvers, B. Groethuysen, H. Norero, La philosophie allemande au XIX siècle, Paris, Alcan, 1912.

36 Remarquons que la conception de Simmel reste sur ce point relativement constante dans ses écrits. Ainsi peut-on lire dans sa 'petite sociologie', parue à la fin de sa vie, que la sociologie est: «(...) un nouveau chemin pour toutes ces (sciences), une méthode de la science, qui justement à cause de sa façon de se tourner vers l'ensemble des problèmes n'est pas une science avec un contenu propre.» (G. Simmel, GSG 16, (1917) 1984, p. 18).

37 Nous ne manquerons pas de revenir sur les différences qui séparent les concepts de société et de sociation chez Simmel, et sur les raisons pour lesquelles Simmel fixe à la sociologie le devoir d'étudier les phénomènes découlant du second, plutôt que du premier concept. 


\section{3. - LA DIMENSION SOCIOLOGIQUE DE LA WECHSELWIRKUNG. ÉTUDE TRANSVERSALE}

Nous appelons la lecture suivante 'transversale' par souci de méthode, et nous justifions ainsi l'emploi et la signification que ce mot prend pour nous.

Il est difficile de comprendre la notion de Wechselwirkung, et ici sa dimension sociologique, en ne recourant qu'à quelques textes principaux de Simmel. La raison en est simple: la notion évolue tout au long de l'œuvre de l'auteur. Pour essayer d'en approcher le cœur, il faut faire des distinctions d'une part entre ce qui est central et ce qui est périphérique dans l'œuvre à ce propos, d'autre part il faut les replacer dans l'évolution générale du concept et de la pensée de Simmel. 'Transversal' suppose donc ces deux significations, qui représentent les deux manières dont nous avons procédé dans cet article pour étudier la Wechselwirkung. Décrivons brièvement notre démarche.

La ligne générale du développement de la Wechselwirkung a été principalement pistée à l'aide des ouvrages suivant: «Über sociale Differenzierung. Sociologische und psychologische Untersuchungen»(1890), Einleitung in die Moralwissenschaften (1892-1893), Philosophie des Geldes (1900), Soziologie (1908), «Grundfragen der Soziologie: Individuum und Gesellschaft» (1917). Ils figurent les tournants principaux de la pensée de Simmel. Ces textes présentent un deuxième avantage non négligeable. Ils recoupent une grande partie des articles que Simmel a écrit ${ }^{38}$, qu'ils aient été reformulés pour tel chapitre de telle ou telle œuvre à venir, ou qu'ils en aient simplement inspirés tels développements. Notre but a donc consisté à chercher l'explicitation de la ligne générale, en mentionnant les boutures originales qui y ont poussé et en ont fait la variété.

Cette première exigence méthodologique s'articule aux quatre registres principaux avec lesquels la pensée de Simmel se confronte pour donner son point de vue sur l'existence humaine. Dans chacun de ces registres, la Wechselwirkung prend une signification particulière, dont nous pouvons suivre le développement tout au long de l'œuvre de l'auteur. En cumulant ces deux dimensions ou significations du mot 'transversal', nous essayons donc d'observer comment la Wechselwirkung se développe et signifie dans l'œuvre de Simmel pour chacun de ses registres particuliers. Nous présentons ici le résultat de l'application de cette méthode dans le cadre de la dimension sociologique.

\section{a) La Wechselwirkung comme lieu de l'objet de la sociologie}

La dimension sociologique de la théorie de Simmel présente l'idée de mouvement, le fondement de la Wechselwirkung, à la manière d'un processus qui fait société. Cette formulation très générale reste toutefois bien insuffisante lorsque nous nous enfonçons dans les textes de l'auteur. Il n'y a pas chez Simmel un seul processus, mais plusieurs qui apparaissent à différents moments dans l'œuvre, et

38 L'exemple le plus frappant est celui que mentionne Rammstedt à propos de la constitution de la Soziologie (GSG 11, (1908) 1992), qui devait être en chantier depuis au moins 1894, et qui rassemble les articles les plus importants produits et retravaillés par Simmel de 1894 à 1908 . A ce propos, voir O. Rammstedt, 1992, pp. 3-21. 
qu'il s'agit d'interroger ${ }^{39}$. Mettons-nous donc en route, en commençant par notre première étape, l'œuvre de la jeunesse de Simmel, «Über sociale Differenzierung. Sociologische und psychologische Untersuchungen» (1890).

Dans ce premier grand texte, Simmel approche la Wechselwirkung de façon indirecte, par le recours au concept de société: «Le concept de société n'a ouvertement un sens que lorsqu'il se tient dans n'importe quel contraire à la simple somme des individualités.»(G. Simmel, GSG 2, (1890) 1989, p. 126) ${ }^{40}$. Cette remarque, lue en référence à la thèse de doctorat sur Kant, n'étonne pas. Pour Simmel en effet, la sociologie n'étudie pas les monades et leur sommation, sur lesquelles se fonderait la signification d'une société. Simmel met plutôt en question à la fois le concept d'individu et celui de société. Pris isolément, ils sont vides de contenus. En tant que tels ils empêchent plus qu'ils ne favorisent la réflexion sociologique parce qu'elle ne peut plus cheminer alors sans tomber dans une absolutisation de l'individu ou de la société. Si ces concepts sont pourtant utilisés par Simmel, c'est comme pôles opposés, comme limites, au sens presque mathématique du terme, permettant de circonscrire le lieu de l'objet de la sociologie dont la formulation première est donnée par la 'Wechselwirkung'. L'objet de la sociologie repose sur et dans ces mouvements dont le spin est en soi porteur d'effets qui occasionnent des changements et des échanges concrets dans le monde. Remarquons toutefois que l'objet de la sociologie n'est pas défini précisément pour autant. C'est, jusqu'ici, le social du fait, une de ses qualités spéciales parmi d'autres (psychologiques, morales, physiques, etc.) qui n'est pas le caractère par excellence en soi et pour soi de tous les phénomènes, ce que Durkheim qualifiait de fait 'sui generis' (E. Durkheim, (1900) 1975, p. 28). Seul son lieu apparaît distinctement, entre les pôles de la société et de l'individu, dans ce balancement de l'un à l'autre que signifie la Wechselwirkung.

Une autre caractérisation de la Wechselwirkung nous est donnée plus loin par Simmel, qui vient compléter ce que nous avons exposé ci-dessus: «Ces éléments ne sont pas par exemple à soi de véritables unités; mais ils sont ici pour traiter de plus haut résumés, parce que chacun a dans son rapport à l'autre un effet unitaire; c'est pourquoi il n'est pas besoin d'être seulement des personnes humaines dont des effets de changements constituent la société, car au contraire il peut s'agir aussi de groupes, qui produisent à nouveau entre eux une société. Il en va aussi de l'atome physique et chimique qui n'est pas un simple Etre au sens de la Métaphysique, mais au contraire pris absolument, quelque chose de toujours à nouveau

39 C'est peut-être notamment pour cela que nous pouvons, comme nous y encourage Vandenberghe, parler plutôt des sociologies de Simmel que de sa sociologie (voir ici F. Vandenberghe, 2000).

40 Ce genre de considération va directement contre certaines interprétations de l'œuvre de Simmel qui tendraient à dire le contraire. Ainsi, Boudon affirme-t-il: «Je laisse ici de côté le fait que la longueur de ce premier chapitre (Boudon parle ici du chapitre des «Problème de la philosophie de l'histoire» (1984)) - qui est consacré au moment microscopique de l'analyse, au moment de la compréhension de l'action - témoigne lui aussi de la perspective individualiste de Simmel.» (R. Boudon, 1998, p. 177). Remarquons que Boudon positive (dans le sens de son optique théorique) et généralise l'approche simmélienne de l'individualité à toute la théorie de Simmel, alors que cela n'en constitue qu'un aspect qui plus est, comme le remarque très bien Vandenberghe (F. Vandenberghe, 1999), n'a rien avoir avec une quelconque revendication préfigurant l'individualisme méthodologique. Il s'inscrit à ce titre dans une tradition de la sur-interprétation de l'œuvre de Simmel, qu'avant lui Durkheim et bien d'autres avaient initiée. 
séparable (...).»(G. Simmel, GSG 2, (1890) 1989, p. 131). En reprenant le contenu de cette citation, les éléments suivant apparaissent. Premièrement, et on peut sans doute y voir un effet de la conception atomiste et positiviste ${ }^{41}$ du jeune Simmel qui donne le ton des premières formulations ayant trait à la Wechselwirkung, sont distingués non plus deux, mais trois pôles ou entités-limites: l'individu, le groupe et la société. Le lieu de la Wechselwirkung s'en trouve élargi, dans la mesure où il est envisagé désormais à partir de trois dimensions sociologiques nécessaires à la compréhension générale du social que cette discipline peut favoriser. Mais au-delà, ceci signifie encore pour Simmel la possibilité de désigner derrière l'individu l'interpersonnel, derrière le groupe l'intergroupal et l'intragroupal, et enfin, derrière la société l'intersociétal et l'intrasociétal.

Ceci étant posé, il est alors évident pour Simmel qu'il y a société, mais aussi individus et groupes, partout où il y a Wechselwirkung: «Où une association a lieu, dont les formes ne lâchent pas prise, même si un seul des membres n'y participe plus ou y pénètre; où une propriété extérieure et commune existe, dont l'achat et la possession n'est pas chose d'un seul; où une somme de connaissances et de contenus moraux de vie existent, qui par la participation d'un seul ne sont ni agrandis ni diminués, qui, devenus pour ainsi dire substantiels, se trouvent prêts pour chacun qui veut les avoir en partage; où le droit, la morale, le commerce des formes est en formation, que chacun mène ou doit mener à terme, et qui pénètre avec les autres un être-ensemble spatial certain - là partout est la société, là grossit l'effet de changement vers un corps, qui se distingue de tel autre comme sociétal, qui disparaît avec le jeu immédiat des sujets à venir et de leurs comportements instantanés.» (G. Simmel, GSG 2, (1890) 1989, pp. 133-134). Sont mises ici en évidence les propriétés fondamentales de la Wechselwirkung relatives à son efficience.

Premièrement, la Wechselwirkung, en tant qu'elle est effet(s) de changement(s), est la société en elle-même. Si nous faisons un pas supplémentaire, nous pouvons logiquement envisager que de la Wechselwirkung découlent les pôles limites qui la circonscrivent, entre lesquelles elle bouge continuellement. Elle contient autrement dit en elle-même les limites de son expansion, en tant que mouvement d'attraction/répulsion, où l'attraction ne peut exister sans la répulsion

41 Cette phase de l'évolution intellectuelle de Simmel est notamment soulignée par Dahme et Rammstedt, Freund et Landmann (H.-J. Dahme, O. Rammstedt, 1984; J. Freund, (1981) 1991; M. Landmann, (1968) 1987). Les auteurs précisent que le positivisme de Simmel n'est pas à comprendre dans le sens par exemple comtien du terme, notamment pour deux raisons. D'une part, par positivisme, il faut plutôt considérer la référence de Simmel à l'évolutionnisme darwinien. De ce point de vue, même s'il y a bien un rapport avec le positivisme des débuts qui reste liés au métaphores biologiques et physiques, il y a distance sur le fond. Le credo de scientificité n'est pas compris chez Simmel dans le même sens que chez Comte, ce que nous laissait déjà deviner la conception simmélienne de la sociologie décrite plus haut. Enfin, il n'y a pas chez Simmel de recherche des lois du social, comme chez Comte. D'autre part, n'oublions pas que l'atomisme, comme la géométrie, la biologie ou la chimie, sont utilisés par Simmel avant tout dans un sens didactique. Par le moyen de ces exemples métaphoriques, Simmel suggère des connexions là où le mot ne permet pas d'aller directement au but, c'est-à-dire risquerait de fermer sur une définition un développement qui se dirige au contraire dans l'ouverture, pour ne pas dire l'éclatement, des définitions. Nous retrouvons ici le style essayiste de Simmel, parfois bien mal compris par certains de ses contemporains (voir par exemple le commentaire de la Soziologie de Simmel par Th.G. Masaryk, 1909; son genre de critique sera repris par Aron (R. Aron, 1935, p. 6)). 
(et inversement) et ne peut donc devenir attraction (ou répulsion) pure, ce qui équivaudrait à sa fixation sur un ou l'autre pôle de cette opposition. C'est là une condition de sa non dispersion (elle est limitée par des pôles) et de sa non réification (elle ne se réduit pas aux pôles), positivement de son caractère de 'mouvement perpétuel' a-synthétique ${ }^{42}$.

Secundo, la Wechselwirkung est au fondement des formes relationnelles qui se produisent entre ces pôles et qui à un moment donné en deviennent indépendantes.

Tertio, la Wechselwirkung se dilue ('Verdünnung'), ce qui a contrario indique qu'un effet de changement isolé ne fait pas société. Cette dilution distingue des niveaux relationnels et donc les combinaisons possibles des pôles entre lesquels ils s'actualisent. Il est en soit l'indice de la différenciation sociale dont parle Simmel dans son ouvrage. Ce processus semble débuter au niveau interpersonnel, situation au fond la plus élémentaire d'une personne en relation avec une ou d'autres personnes. Simmel le privilégie, comme il nous le rappelle ici: « $\mathrm{Au}$ contraire du mouvement de tout le groupe, qui se présente à la pensée sociologique comme le prochain objet, les réflexions suivantes doivent dessiner dans l'essentiel la position et le destin de l'individuel, comment il se préparera à travers tel effet de changement avec les autres, qui l'unit avec ces autres à un tout social.» (G. Simmel, GSG 2, (1890) 1989, p. 138).

Cette première mise au point permet d'engager une critique de l'épistémologie classique, expression qui se réfère chez Simmel de manière privilégiée à la théorie de la connaissance kantienne. Ceci se produit dans l'Einleitung deux ans plus tard.

\section{b) Wechselwirkung et critique de l'épistémologie classique.}

L'Einleitung in die Moralwissenschaften (1892-1893) présente un prolongement de la réflexion amorcée dans «Über sociale Differenzierung» sur la Wechselwirkung, qui passe par une critique des principaux concepts de la philosophie.

Conséquemment, et comme l'avait déjà souligné Bouglé, l'auteur ne se soucie pas vraiment dans ce livre de définir une science morale. Il se contente d'appliquer vingt définitions au Devoir, au Bonheur, à la Liberté, à la Responsabilité, à l’Unité et à la Multiplicité des fins... Il désire «[...] établir qu'ils (ces concepts)

42 Ce que nous voulons en particulier souligner par cette expression est le caractère, dialectique sans doute, mais non-hégélien de ce mouvement des Wechselwirkungen. Il n'y a pas de 'Aufhebung' chez Simmel, comme il n'y a pas la réunion des pré-conditions nécessaires à la 'Aufhebung' hégélienne, à savoir la possibilité pour les pôles de se concilier dans une synthèse supérieur qui est en même temps autre chose que cette bipolarisation. Les pôles restent chez Simmel inconciliables ('unversöhnlich'; Simmel en parle à plusieurs reprises dans son œuvre, et notamment dans Soziologie (G. Simmel, GSG 11, (1908) 1992, pp. 71, 173, 309-311) et Philosophie des Geldes (G. Simmel, GSG 6, (1900) 1996, p. 504)), et le mouvement dialectique des Wechselwirkungen ne parvient jamais à la synthèse. Ce principe de dualité opérant l'a-synthèse est relevé à juste titre dans toute son importance par Landmann (M. Landmann, 1976). C'est finalement une nuance qui pourrait s'exprimer ainsi: si pour Hegel le réel est rationnel et le rationnel est réel, pour Simmel, le réel est relationnel et le relationnel est réel. Par rapport à la dialectique hégélienne, la Wechselwirkung représente un décentrement vis-à-vis de la 'Aufhebung' (comme vis-à-vis du formalisme et de l'esprit objectif). Elle est un autrement de la dialectique hégélienne et finalement, l'autre de cette dialectique, ce qui prendra chez Simmel le nom de 'Leben' (Vie). 
sont indéfinissables, et leur contour indéterminé. (...) La spéculation sur le Devoir et l'Etre est impuissante à déterminer les objets de nos représentations et de nos actions; car le Devoir et l'Etre n'entrent pas dans la série de ces objets, mais seulement dans la série des formes psychologiques, que l'expérience peut adapter à toutes les matières. Chacune de nos représentations comprend, en effet, deux éléments: l'objet, puis le sentiment qui l'accompagne, qui nous dit, par exemple, si l'objet est réel ou seulement idéal.» (C. Bouglé, 1912, pp. 190-191). Devoir, Etre, etc. sont des formes dans lesquels les philosophes expriment des contenus de vie pour Simmel. En tant que tels, ces concepts échappent à l'existence propre des philosophes et des philosophies qui les ont produits. Il en découle trois conséquences pour notre auteur.

Premièrement, les concepts analysés, devenus autonomes des individus, constituent une 'culture objective' ${ }^{43}$, un ensemble de formes, qui s'opposent à la vie, puisqu'ils en fixent les moments plutôt qu'ils ne parviennent à en comprendre la dynamique. Il sont donc impropres à en rendre les propriétés immanentes, et ne peuvent nous en donner qu'une représentation statique et très contextualisée.

Deuxièmement, en tant que forme, ces concepts reçoivent les contenus les plus variés. Se pose donc la question de savoir ce que signifie le fait de se polariser sur l'un ou l'autre de ces contenus, et quelle est la légitimité d'un tel positionnement.

D'autant plus que, et troisièmement, un seul contenu peut également prendre des formes variées.

Le problème que soulève Simmel est celui du caractère à la fois contraignant et non homogène a priori du concept qui, pour cette raison, ne peut vraiment saisir le mouvement des Wechselwirkungen. Simmel ne va pas seulement le remarquer, dans une intention qui a semblé à certains être 'déconstructioniste' ${ }^{44}$. Il désigne en plus l'autre lieu de la réflexion des sciences humaines: le processus. En pensant le processus, et plus concrètement le relationnel, nos concepts et la méthode qu'ils supposent montrent leurs limites immanentes. Ceci force les sciences humaines soit à se contenter d'une représentation appauvrie et unilatérale de la 'réalité', soit à développer une autre perspective. La première solution nous conduit aux problèmes que soulève Simmel dans son Einleitung, à savoir l'impossibilité de fonder une perspective autrement que sur le dualisme ou le monisme, que sur l'individualisme ou le holisme, que sur le nominalisme ou le rationalisme, etc. Simmel tente donc le second chemin; il reprend et approfondit tout d'abord la conception générale de la Wechselwirkung esquissée dans «Über sociale Differenzierung».

43 Nous retrouvons la définition que Simmel donne de la culture objective et de la culture subjective dans un texte de 1908, «Vom Wesen der Kultur»: «On peut décrire la culture objective comme étant les choses dans chaque élaboration, dans chaque amélioration, dans chaque complétude, avec laquelle elles mènent l'âme à quelque complétude ou qui représentent les chemins, qui conduisent le seul ou la totalité sur la route d'une présence plus précise. Sous culture subjective je comprends la mesure atteinte du développement de la personne (...).» (G. Simmel, GSG 8, (1908) 1984, p. 90). En d'autres termes, la culture objective représente l'objectivation de soi dans des formes de sociation, quant à la culture subjective, elle traduit le mouvement complémentaire inverse de subjectivation de ces mêmes formes.

44 Voir sur ce point Frank (A. Frank, 1987). Dans un sens analogue, on entend dire que Simmel est un pré-postmoderne (D. Weinstein, M.A. Weinstein, 1993). Il s'agit à nouveau d'une exagération avec laquelle nous ne saurions nous accorder, tant elle manifeste d'une lecture rétrospective et exagérée du problème des polarités simméliennes. 
Il affirme à nouveau: «[...] là partout est la société, là s'engrosse l'effet de changement vers un corps, qui se distingue de tel autre comme sociétal, qui disparaît avec le jeu immédiat des sujets à venir et de leurs comportements instantanés. Ici, l'effet de changement a atteint tel degré d'intensité, qui laisse apparaître les individus dans leur ensemble comme une unité, et qui dans la relation indécidable élève l'individu en tant que tel.» (G. Simmel, GSG 3, (1892) 1989, p. 165). Le dernier membre de phrase ajoute deux éléments supplémentaires à ceux relevés dans «Über sociale Differenzierung». D’une part, la Wechselwirkung est présentée comme une relation indécidable et d'intensité variable entre des individus dont elle contribue d'autre part à forger et à faire se révéler la personnalité. L'indécidabilité de la Wechselwirkung est le signe de son indépendance relative vis-àvis de l'individu. Cette indépendance est unilatérale. L'individu ne peut échapper à la Wechselwirkung, il est touché par son mouvement de l'extérieur et de l'intérieur. Par contre, la Wechselwirkung, qui se cristallise dans des formes, lui échappe. C'est une première compréhension de l'indécidabilité de la Wechselwirkung et de toute relation humaine. Mais nous pouvons y repérer encore une seconde, qui est le complément de la première: les individus, groupes ou sociétés en relation ne peuvent décider du processus en lui-même comme nous l'avons vu. Autrement dit, la Wechselwirkung contient son sens, son but et son effectivité en elle-même. En ce dernier sens donc, l'indécidabilité de la Wechselwirkung permet logiquement à Simmel d'en proclamer l'ouverture infinie, c'est-à-dire la perpétuité de sa dynamique de balancement. En réunissant ces deux compréhension de l'indécidabilité, nous sommes amenés à constater qu'elle garantit à Simmel la non-inscription du mouvement de la Wechselwirkung dans un idéalisme théorique, qu'il soit transcendantal, ontologique ou organique, et en même temps évite le pragmatisme décisionnel-individuel ${ }^{45}$. Rien ne permet encore pour l'instant de préciser la signification de ces transitions du particulier à l'universel ou de l'individu au tout. Mais force est de constater que l'inscription de ces problématiques dans l'idée de Wechselwirkung désigne un autre lieu de la réflexion sur ce que nous appelons 'société' et 'individu', à savoir: les relations sociales.

Simmel parle également de l'intensité de la Wechselwirkung. Comme nous l'avons vu plus haut dans «Über sociale Differenzierung», la Wechselwirkung permet de différentier des niveaux processuels en individuels, groupaux, sociétaux. L'intensité nous permet de préciser que nous passons d'un niveaux à l'autre selon la fréquence des Wechselwirkungen. La fréquence des Wechselwirkungen distingue donc des processus correspondant à des niveaux sociologiques et à des pôles relationnels différents.

Conséquemment, le résultat que nous livre l'Einleitung peut être résumé ainsi:

1. Simmel critique l'épistémologie classique à partir de l'idée de processus, et tente d'ouvrir une autre voie épistémologique basée sur la Wechselwirkung;

2. par suite, il précise le sens de la Wechselwirkung; c'est un processus qui a un degré d'indécidabilité, et qui est en ce sens indépendant des termes entre lesquels il a lieu; par contre, la réciproque est fausse, puisque les entités qui fixent

45 C'est la démonstration par excellence critique de la rationalité historique que redoublera Simmel à propos de l'histoire dans les «Probleme der Geschichtsphilosophie» (GSG 2, (1893) 1989). 
les limites de la Wechselwirkung dépendent de ce processus; enfin, la Wechselwirkung possède une intensité qui permet de distinguer les différents processus que nous pouvons obtenir selon les niveaux sociologiques mis en évidence.

Nous observons donc que les charnières centrales de l'épistémologie sociologique de Simmel se mettent en place en même temps que le sens du mot Wechselwirkung se précise. Corollairement, la Wechselwirkung ouvre à de nouvelles dimensions proprement psychosociologiques ${ }^{46}$ et métaphysiques de par les propriétés d'indécidabilité et de fréquence dont elle est dotée. En effet, à partir du moment où le relationnel déborde l'individualité/la société au sens classique de ces termes, deux questions se posent: 1) que signifie ce débordement et 2) comment l'individualité/la société en tant que processus, donc en tant qu'individuation/sociation, peuvent-ils être compris? Dans le contexte où nous nous trouvons actuellement, ce qui importe toutefois est surtout la distinction que Simmel fait entre la logique interne du fonctionnement individuel/groupal/sociétal et la logique des relations sociales. Si les hommes et les sociétés répondent de quelque chose qui leur est propre, d'un gradient de vérité immanent à leur vie, d'une 'loi individuelle ${ }^{47}$, il en va de façon analogue pour les relations sociales. Il y a donc une étude scientifique possible des relations sociales.

Cette hypothèse que porte l'Einleitung est reprise dans l'article célèbre que Simmel publie en 1894: «Das Problem der Sociologie». C'est un moment charnière pour l'auteur. Il clôt la critique que proposait l'Einleitung pour ouvrir une programmatique qui n'a d'autres ambitions que de fonder la sociologie ${ }^{48}$. Examinons ceci de plus près.

\section{c. Wechselwirkung, Vergesellschaftung, Tausch. Programme pour une sociologie scientifique.}

Dans «Das Problem der Sociologie», Simmel met en rapport la Wechselwirkung avec un concept qui n'était pas apparu jusqu'ici, la 'Vergesellschaftung', que nous pouvons traduire presque littéralement par 'faire société' ou sociation"

46 Nous retrouvons ici l'interprétation que Bouglé donne de l' Einleitung in die Moralwissenschaften (1892-1893) : «Il (Simmel) confesse, ou plutôt il proclame que les interactions qui lui paraissent être les éléments constitutifs de la vie sociale sont des phénomènes psychologiques. Et pour lui comme pour Tarde (...) l'analyse des interactions mentales est l'essentiel de la sociologie. (...) Par où l'on mesure à quel point sa pensée, si elle se rapproche de celle de Tarde, s'éloigne de celle de Durkheim.» (C. Bouglé, 1912, p. 202). On retrouve également des remarques allant dans ce sens chez Freund (J. Freund, (1981) 1991), en particulier au sujet du relativisme de Simmel.

47 Voir notamment à ce sujet le recueil parut sous ce titre: Das individuelle Gesetz. Philosophische Exkurse ((1968) 1987). On peut également trouver l'exemple par excellence de la loi individuelle chez Simmel, qu'il nous livre à l'occasion de la préface qu'il réalise pour les œuvres complètes de Goethe, dont le texte est écrit aux alentours de 1917-1918.

48 Il revient à Rammstedt (O. Rammstedt, 1991; 1992) d'avoir montré toute l'importance de cet article pour la fondation de la sociologie de Simmel.

49 Nous choisissons sciemment de ne pas traduire ce terme par 'socialisation', comme cela a pu être fait dans le passé (voir notamment N. Spykman, 1925) et même le présent (voir notamment la traduction française de la Soziologie de Simmel par L. Deroche-Gurcel et S. Muller Sociologie: Étude sur les formes de la socialisation, Paris, PUF, 1999). La socialisation désigne traditionnel- 
«[...] que le succès de ces causes, la stimulation de ces buts mènent à un effet de changement, à une sociation de ses porteurs, c'est une forme, dans laquelle chaque contenu se vêt et dont la séparation de la dernière abstraction scientifique possible repose sur toute l'existence d'une science spéciale de la société.» (G. Simmel, GSG 5, (1894) 1992, p. 54). Il y a, dans cette citation, une identité presque totale entre sociation et Wechselwirkung. Mais il y a en même temps l'expression d'une différence de niveau entre eux, rappelée en début et en fin de citation. La Wechselwirkung est utilisée pour parler de relations sociales particulières, à la limite seulement interpersonnelles. La sociation, composé d'une masse consistante de Wechselwirkungen, se situe au niveau d'un processus global de 'faire société'. Simmel différentie donc deux processus sociaux (Wechselwirkung et Vergesellschaftung) et deux niveaux heuristiques d'une sociologie, sans les séparer radicalement. Cette différentiation, apparemment flottante ${ }^{50}$, en fait graduelle $^{51}$, renforce la relativisation du concept de société, et en constitue à la fois une critique interne. Au fond, semble nous suggérer Simmel fidèle en cela à son Einleitung, il ne peut y avoir de 'société' qu'à l'état de concept. C'est pourquoi, parler de société ne peut se faire qu'entre guillemets ${ }^{52}$. La société qui existe dans le concret de nos vies quotidiennes n'est autre que la sociation née de la fréquence des Wechselwirkungen que des individus (groupés ou non) 'mettent en œuvre' ou 'portent'. Ces éléments supplémentaires apportés à l'élaboration du contexte sociologique de la Wechselwirkung vont être retravaillés et prolongés dans cet écrit célèbre qu'est Philosophie des Geldes (1900).

Simmel rappelle d'abord le rapport étroit qui lie la Wechselwirkung à l'interpersonnalité: «[...] nous ne pouvons nous représenter comme le point de départ de toute silhouette sociale que l'effet de changement de personne à personne.» (G. Simmel, GSG 6, (1900) 1996, p. 208). Le contexte interpersonnel incluant les actions visant un but, la Wechselwirkung est spécifiée comme leur lieu privilégié: «La signification principale de l'agir en vue d'un but se trouve ainsi dans l'effet de changement, qui le fonde entre le sujet et l'objet.» (G. Simmel, GSG 6, (1900)

lement l'acquisition d'une expérience de vie à travers les relations qu'un individu entretient à d'autre individus, des groupes ou des sociétés. Le 'faire société' se situe à un niveau épistémologique plus général et plus abstrait, qui comprend la socialisation, mais qui traduit un mouvement d'ensemble, un devenir société des relations que les individus et les groupes entretiennent entre eux. C'est pourquoi nous proposons le terme 'sociation' qui donne une signification peut-être plus appropriée à ce qu'évoque le 'faire société'. Remarquons également qu'en cela nous nous trouvons en accord avec la traduction déjà proposée par Bertrand Chokrane dans la publication de la traduction française du chapitre 7 de la Soziologie de Simmel (B. Chokran, Les pauvres, Paris, Quadrige/PUF, 1998).

50 Il n'est pas difficile de voir ici que Simmel ne travaille pas la question de la teneur épistémologique des concepts qu'il mentionne. Ainsi, nous ne savons pas si la Wechselwirkung est plus générale ou abstraite que la Vergesellschaftung, ou si c'est le contraire. En fait, la question se résout en posant la caractérisation des concepts de façon correspondante aux niveaux épistémologiques à partir desquels ils sont décrits. D'un point de vue analytique, la Wechselwirkung est plus abstraite que la Vergesellschaftung; mais d'un point de vue pratique, ou 'synthétique' comme dit Simmel dans sa Philosophie des Geldes, la Wechselwirkung est le particulier, et la Vergesellschaftung le général.

51 La Wechselwirkung comme concept graduel ('gradueller Begriff') signifie que la Wechselwirkung varie en étendue et en intensité. Nous développons plus bas ces deux caractéristiques.

52 C'est d'ailleurs ce que fait Simmel presque tout au long de son œuvre. 
1996, p. 256). Simmel va même plus loin en identifiant le processus des actions effectuées en vue d'un but à la Wechselwirkung: «[...] le processus en vue d'un but signifie un effet de changement entre un Je personnel et ce qui lui est nature extérieure.» (G. Simmel, GSG 6, (1900) 1996, p. 258). Ces arguments ajoutent encore au statut sociologique de la Wechselwirkung. Elle se situe entre le sujet et l'objet, le Je et la Nature. Elle se généralise, s'étend pour ne plus signifier seulement un rapport entre personnes, mais encore un rapport entre l'homme et le monde qui l'entoure. Elle débouche corrélativement sur un nouveau processus, l'échange: «On doit être clair sur ce point, que la pluralité des relations des hommes entre eux peut valoir comme échange; il est en même temps effet de changement pur et montant, qui de son côté résout la vie humaine, aussitôt qu'elle veut gagner une matière et un contenu. (...) Chaque effet de changement doit être considéré comme un échange: chaque entretien, chaque amour (...), chaque jeu, chaque regard à soi.»(G. Simmel, GSG 6, (1900) 1996, p. 59). Le concept d'échange ('Tausch') ${ }^{53}$, comme c'était le cas pour la sociation, se distingue sans se séparer de la Wechselwirkung. Il prend la signification d'un mouvement global des Wechselwirkungen, autrement dit de son état fréquentiel maximal, dont la fonction est de réaliser cette société dans et par la connexion interne des relations sociales: «La fonction de l'échange, un effet de changement immédiat entre individus, est cristallisée avec lui à travers un objet constitué pour soi $(\ldots) ;(\ldots)$ l'échange lui même est une des fonctions qui apporte hors du simple être-à-côté des individus leur connexion interne, la société, à la réussite; car la société n'est pas une unité absolue, qui devrait seulement être là, avec laquelle toutes les seules relations de ses membres: sur- et sous-ordre, cohésion, imitation, division du travail, échange, attaque et défense également dirigées, communauté religieuse, formation de parti et beaucoup d'autre se développeraient en elle en tant que porteurs ou cadres de chacun. (...) C'est pourquoi il y a presque une double expression à dire que l'échange effectue la sociation: il est bien plus qu'une sociation, que l'une quelconque des relations, dont la composition mène la somme des individus à un groupe social, parce que 'société' est identique à la somme de ces relations.»(G. Simmel, GSG 6, (1900) 1996, pp. 209-210). De ce point de vue,

53 Si l'idée d'échange est présente dès le départ chez Simmel dans sa conception du mouvement et de la Wechselwirkung en général, le concept lui-même apparaît plus tard. Nous en repérons, semble-t-il, les premières occurrences dès le texte de 1895 «Zur Soziologie der Familie». Simmel mentionne le concept d'échange dans le contexte suivant: «Qu'une femme se vende, ne serait ce que dans le mariage avec celui qui lui serait un mari valable, ne serait-ce qu'en des formes fuyantes, nous apparaît cependant tellement particulièrement contraire, parce que avec ceci le tout personnel, ce que l'homme a à donner, est donné dans l'échange avec une valeur impersonnelle, comme s'il s'agissait d'argent.» (G. Simmel, GSG 5, (1895) 1992, p. 89). Cette citation anticipe sur le propos que Simmel tiendra dans Philosophie des Geldes. Elle attire en outre l'attention sur un élément que nous retrouvons à l'occasion de nos analyses: le concept d'échange est presque chaque fois mentionné par Simmel en rapport avec un propos sur l'argent. Il n'en garde pas moins une signification qui, en vertu de son lien à la Wechselwirkung, ne se limite pas à celle de l'échange économique, voire n'y réfère pas. On se souviendra à ce propos des premiers mots de Simmel dans sa Philosophie des Geldes: «Pas une ligne de ces recherches est pensées dans le sens de l'économie nationale. Ce qui veut dire, que l'apparition de la valeur et de l'achat, de l'échange et du moyen d'échange, des formes de production et des valeurs de la fortune que l'économie nationale ne considère que d'un point de vue, sont considérés ici d'une autre point de vue » $(\mathrm{G}$. Simmel, GSG 6, (1900) 1996, p. 11). 
l'échange doit être considéré par le sociologue comme le concept de société le plus global et le plus concret à la fois, car il est ce que le mot société veut dire, à savoir son contenu de vérité actualisé diversement dans le quotidien humain.

Nous voyons donc que Simmel utilise, sur la base et en relation avec la Wechselwirkung, deux processus supplémentaires/complémentaires qu'il nomme sociation et échange. Bien qu'ils s'en détachent relativement, ils représentent deux moments différents de l'extension de la Wechselwirkung, et ses deux fonctions principales, à savoir:

1. le 'faire société' ou sociation, qui est le processus même d'effectuation et d'extension des relations sociales, c'est-à-dire de leur augmentation fréquentielle (il y a plus de relations sociales);

2. la réalisation du 'faire société' ou échange, à savoir l'extension et la concrétisation des sociations, autrement dit la 'société' (il y a plus que des relations sociales, il y a une 'société').

Philosophie des Geldes permet donc de distinguer trois niveaux épistémologiques de la Wechselwirkung, en allant de l'abstrait (mouvement/Wechselwirkung) au concret (échange) par l'intermédiaire d'une transition (sociation/effectuation-augmentation des Wechselwirkungen). Cette première conception est redoublée d'une seconde qui la complète, où la Wechselwirkung apparaît comme le particulier, et l'échange le général, avec ce stade intermédiaire de l'un à l'autre que représente la sociation.

Tableau 1. Wechselwirkung, Vergesellschaftung et Tausch.

\begin{tabular}{|lcc|}
\hline & Ordre des processus & Ordre de la concrétisation de la société \\
\hline Wechselwirkung & Particulier & Abstrait \\
Vergesellschaftung & Transition & Transition \\
Tausch & Concret & Général \\
\hline
\end{tabular}

Cette conception chiasmatique de la Wechselwirkung ne variera pas vraiment chez Simmel jusqu'à sa Soziologie (1908), où elle semble se structurer plus fortement. Voyons comment ceci se produit.

Soziologie peut être interprétée comme l'écrit qui assoit le plus les acquis de la réflexion de Simmel sur la Wechselwirkung accumulés jusque là. Nous y trouvons, comme c'était déjà le cas dans Philosophie des Geldes, une mise entre parenthèses de la teneur psychologique qu'évoque le concept ${ }^{54}$, pour en accentuer corrélativement le côté sociologique. Simmel répète que la Wechselwirkung s'étend aux sens de la sociation et de l'échange. Soziologie reprend également le problème de la frontière de la Wechselwirkung, que nous avions déjà mentionné plus haut, et qui est ici explicité: «[...] l'unité d'effet de changement, la relation

54 Simmel renoue sur ce point avec la métaphore atomiste, inspirée par Schäfle, en préférant parler d'atomes plus que d'individus: «Ici reposent celles qui portent seulement les microscopies psychologiques entrant dans les effets de changements entre les atomes de la société, celles qui portent toutes les duretés et les élasticités, toute la coloration et l'unité de cette vie de la société si claire et si secrète.» (G. Simmel, GSG 11, (1908) 1992, p. 33). 
fonctionnelle de chaque élément à chaque autre gagne son expression spatiale dans les frontières cadrantes.»(G. Simmel, GSG 11, (1908) 1992, p. 694). Cette idée est reprise et approfondie plus loin: «[...] la frontière sociologique, signifie un effet de changement en soi. Chacun des deux éléments a un effet sur l'autre, dans lequel s'y tient une frontière, mais le contenu de cet effet a justement la caractéristique de n'absolument pas vouloir ou pouvoir avoir de l'effet au-delà de la frontière, et donc aussi sur l'autre.» (G. Simmel, GSG 11, (1908) 1992, p. 697). La frontière n'a pas d'existence indépendamment de la Wechselwirkung. Elle en est en même temps la limite immanente. Caractériser cette limite revient à dire que la Wechselwirkung ne transforme pas de fond en comble l'individu. Elle ne l'atteint pas au plus profond de ce qu'il est. Nous retrouvons ici l'affirmation implicite du principe relativiste de distinction entre le domaine de l'individuel et du relationnel, de la 'loi individuelle' et de la 'loi relationnelle', entre-aperçu plus haut. Il nous est rappelé plus loin, lorsque Simmel aborde, à titre exemplaire, la signification de la Wechselwirkung comme don: «Car le don n'est en aucun cas seulement un effet léger de l'un sur l'autre, mais au contraire il est justement ce qui est contraint par la fonction sociologique: il est effet de changement. A travers l'autre accepté ou refusé, il exerce un effet de retour tout à fait précis sur le premier. La façon qu'il a de l'accepter, avec ou sans reconnaissance, ainsi ce qu'il a déjà attendu ou ce qui l'a surpris, ainsi ce qui l'a satisfait du don ou ce qui reste insatisfait, ainsi ce qu'il a senti élevé ou amoindri par le don - tout cela exerce quelque chose de très décidé, même si naturellement ce n'est pas en concepts précis et en masse de retour exprimable à ce qui a été donné, et ainsi chaque don est un effet de changement entre le donateur et le récepteur.» (G. Simmel, GSG 11, (1908) 1992, p. 663). Le don est cet effet de changement qui ne modifie pas le sens profond des hommes, du donné, ou du recevoir, mais qui fait effet sur les hommes ou les relations qu'ils ont entre eux. C'est seulement dans cette mesure que la Wechselwirkung est le monde du possible de l'humain. A travers elle, chaque individu et chacune de ses actions trouvent simultanément leur possibilité, leur limite, leur développement et leur actualisation. Ces quatre aspects de la Wechselwirkung résument la conception sociologique que nous en donne Simmel dans sa Soziologie. Ils concentrent l'expression humaine toute entière et la remettent au fondement. La Wechselwirkung y apparaît comme mouvement (attraction/répulsion $)^{55}$, richesse (grossissement) et mesure (frontière) concrétisable (échange) et concrétisée dans les relations sociales quotidiennes.

De façon surprenante, Simmel ne va plus revenir sur ces développements. Il ne va pas donner plus de poids à la Wechselwirkung dans l'aspect sociologique de son œuvre. Nous repérons ceci à travers trois modifications importantes de son propos.

Premièrement, le concept d'échange élaboré au cours de Philosophie des Geldes et affermi dans Soziologie disparaît presque complètement des textes de l'auteur, pour être repris, d'ailleurs très ponctuellement, en 1917 dans les «Grundfragen der Soziologie: Individuum und Gesellschaft». Ne reste comme processus à côté de la Wechselwirkung que la sociation.

55 Soulignons que c'est dans sa Soziologie que Simmel montre l'importance de l'attraction/répulsion. Elle est ce qui permet à la Wechselwirkung de prendre ensuite forme: «Comme le cosmos 'Amour et Haine' a besoin de forces attractives et répulsives pour avoir une forme, la société a besoin également d'une certaine quantité de rapports d'harmonie et de disharmonie, d'association et de concurrence, de faveur et de défaveur pour acquérir une forme précise.» (G. Simmel, GSG 11, (1908) 1992, p. 286). 
Deuxièmement, Simmel accentue la teneur psychologique de la Wechselwirkung en y revenant par l'intermédiaire d'un discours sur les groupes et les individus. Wechselwirkung et sociation retrouvent par là-même un signification distincte, qui permet de les concevoir à nouveau comme deux niveaux heuristiques différents, bien que non séparables strictement.

Troisièmement, il abandonne l'idée de Wechselwirkung comme frontière et il en propose une conception plus métaphysique. C'est ce qui apparaît de façon frappante dans sa 'petite sociologie', les «Grundfragen». Ceci est toutefois déjà en germe dans le passage de la Philosophie des Geldes à la Soziologie. C'est ce tournant que nous présentons brièvement maintenant, tel qu'il s'articule à l'aspect proprement sociologique de la réflexion de Simmel.

\section{d) De la sociologie à la métaphysique. \\ Le tournant du développement de la dimension sociologique de la Wechselwirkung}

Le tournant de la dimension sociologique de la Wechselwirkung vers le registre métaphysique se manifeste directement et explicitement dans le texte de 1906, «Die Religion». Simmel affirme: «D'un effet de changement lui-même, il ne peut plus en être question dans ce discours. Son unité métaphysique et substantielle est ce que possèdent directement, non pas l'organisme ou la société, mais les membres comme échange de leur énergies.» (G. Simmel, GSG 10, (1906) 1995, p. 104). Jamais la Wechselwirkung n'avait été caractérisée ainsi à notre connaissance avant 1900. Se développe dans le moment même où semble s'affermir le programme sociologique de Simmel une autre orientation que l'auteur va finalement poursuivre jusqu'à la fin de ses jours. Cette orientation est celle de la philosophie de la vie ('Lebensphilosophie'). Après avoir été fortement sociologue, bien que tenant à son titre de philosophe, après avoir montré très tôt, dans l'Einleitung, que la philosophie ne pouvait être justement qu'une méthode propre à guider le penseur, Simmel renverse la perspective. C'est la philosophie qui revient dans le discours sociologique. C'est cette perspective que les «Grundfragen» développent d'entrée de jeu: «Mais encore est-il d'un tout autre point de vue d'avouer que l'existence humaine n'est véritable que sur la base des individus, sans qu'en souffre la validité du concept de société. Le résume-t-on dans sa plus lointaine généralité, ainsi signifie-t-il l'effet de changement spirituel entre individus.»(G. Simmel, GSG 16, (1917) 1984, pp. 11-12). La Wechselwirkung devient ici 'spirituelle', à savoir essentiellement traitée du point de vue de l'abstraction qu'elle désigne comme mouvement généralisé, comme relationnalité dès lors totale et plus seulement sociale. Ceci ne va pas sans affecter les concepts qui lui sont directement liés, à savoir la sociation et l'échange.

L'effet de l'absolutisation de la Wechselwirkung en principe métaphysique ${ }^{56}$ a pour conséquence un flottement généralisé des concepts de sociation et d'échange. Certes, ces concepts conservent la signification générale qu'ils ont: ce

56 Simmel le précise lui-même dans son autoportrait inachevé «Aus einer Selbstdarstellung», qui devait résumer en une demi page sa pensée: «De cette signification sociologique du concept de Wechselwirkung s'élève pour moi progressivement un principe métaphysique par excellence.» (G. Simmel, (1917) 1997, p. 181). 
sont des manifestations de la Wechselwirkung. Il n'en subissent pas moins le contre-coup de l'élaboration abstraite qui touche la Wechselwirkung. Ainsi par exemple, la sociation apparaît toujours en lien avec la fréquence ou le grossissement des Wechselwirkungen: «Seul est ici l'effet de changement également si superficiel et volant, qu'on pourrait parler dans sa masse de sociation, en pensant que de tels effets de changement deviennent seulement plus fréquents et intensifs dans le besoin de s'associer à plusieurs autres généralement comparables pour justifier cette description.» (G. Simmel, GSG 16, (1917) 1984, p. 12). Mais désormais, elle se particularise de la Wechselwirkung en représentant une sorte de cas pur, stylisé: «Si tant est que la sociation est un effet de changement, il en va ainsi des cas les plus purs et pour ainsi dire les plus stylisés, quand elle s'associe avec d'autres semblables, comme la symétrie et l'équivalence sont les formes de stylisation artistiques les plus claires des éléments perceptibles.»(G. Simmel, GSG 16, (1917) 1984, p. 58). Enfin, l'échange n'est plus mentionné que ponctuellement.

Comment ceci est-il possible? Simmel aurait en effet pu diriger ses recherches vers l'approfondissement des processus de sociation, et globalement l'étude systématique du passage de la Wechselwirkung à l'échange. Or il ne le fait pas, bien qu'il s'en propose le plan: «Peut-on dire, la société serait l'effet de changement entre individus, alors le devoir de la science de la société dans le sens le plus strict et le plus véritable du mot 'société' serait de décrire les formes de cet effet de changement.» (G. Simmel, GSG 16, (1917) 1984, p. 27). Il faut se rendre à l'évidence, Simmel nous laisse cette proposition sans s'y atteler lui-même systématiquement. La fin de sa carrière et de sa vie est marquée de cette orientation nouvelle, métaphysique dans son ensemble, qui culminera dans sa «Lebensanschaung» (GSG 16,(1917) 1998). Nous laisserons toutefois ce point en suspens pour d'autres investigations; concluons pour le moment sur la dimension sociologique de la Wechselwirkung.

\section{4. - SYNTHÈSE: LE REGISTRE SOCIOLOGIQUE DE LA WECHSELWIRKUNG. RELATIONNISME ET RELATIVISME}

Au terme de notre parcours dans le contexte sociologique de la Wechselwirkung chez Simmel, nous pouvons dégager trois phases qui résument les articulations principales de sa réflexion.

La première phase va des premiers écrits sociologiques qu'inaugure la critique de Kant aux dernières années du XIX ${ }^{\mathrm{e}}$ siècle. L'auteur développe la Wechselwirkung dans le sens d'un mouvement d'attraction/répulsion entre humains, ce qui lui permet dans un premier temps de mettre en évidence l'idée de processus. Cette idée lui permet d'adresser une critique à l'épistémologie classique, et de proposer des critères méthodologiques de base permettant de fonder un programme de sociologie scientifique. Ces critères, affirmés de façon presque dogmatique en 1894, prennent racines dans la perspective relationnelle de Simmel, et permettent de distinguer les niveaux analytiques des différents processus que la sociologie doit étudier.

La seconde phase comprend les écrits que Simmel produit entre 1898/1900 et 1906/1908 environ. La Wechselwirkung est présentée comme le niveau abstrait et 
particulier d'un processus dont se distinguent sans s'en séparer deux autres processus, à savoir la sociation et l'échange. La sociation est le processus de 'faire société' en tant que tel. Il induit l'établissement de rapports sociaux et les variations de leur extension fréquentielle. L'échange désigne le processus concret et général de sociation; il est ce que Simmel nomme vers 1908 la 'société' concrète en tant que relations. A travers la distinction de ces deux processus, Simmel approfondit également les propriétés immanentes de la Wechselwirkung qu'il avait seulement esquissées jusque là. Ce sont le mouvement, la richesse et la mesure des Wechselwirkungen. Nous retrouvons ici, sous trois concepts différents, les qualités fondamentales de toutes relations sociales pour Simmel.

La dernière phase illustre les écrits de Simmel parus après 1908, et dont l'origine se situe ou apparaît déjà au milieu de la seconde phase évoquée ci-dessus, attestée notamment par sa sociologie de la religion (1906). Simmel absolutise progressivement la Wechselwirkung, qu'il définit à la fin de sa vie comme un principe métaphysique. Par conséquent, les concepts principaux qui s'y rattachent, en particulier la sociation, subissent également une absolutisation relative. La sociation est perceptible comme style relationnel pur. Enfin, l'échange n'est plus vraiment repris sous la plume de l'auteur. Les fines distinctions cèdent la place à une voie plus globale, une forme d'anthropologie philosophique.

Reste à comprendre cette ambivalence qui tient au double mouvement que l'on voit se développer dans l'œuvre de Simmel, à savoir celui de la fondation d'un relativisme sur la relationnalité des Wechselwirkungen comme processus de concrétisation de la société en tant que relations sociales, et celui de la montée en abstraction de ce relativisme même, qui signifie en même temps sa généralisation absolue. Becher nous apporte un élément de réflexion éclairant lorsqu'il affirme: «Le concept de Wechselwirkung est à cause de sa dynamique déjà un concept de la vie.» (H.J. Becher, 1971, p. 21). En effet, Simmel, lorsqu'il s'intéresse à la sociologie vers 1886 , cherche avant tout à dissoudre la tentative idéaliste d'établir les concepts philosophiques et moraux sur la base de 'lois' de comportements. Il est parti d'une critique de la Völkerpsychologie de Steinthal, et de l'idéologie optimiste du progrès de Spencer et de Comte avec des concepts à connotation darwinienne. Ainsi en va-t-il du concept de différentiation sociale. Mais il ne faut pas oublier que ce darwinisme a semblé à Simmel la seule façon d'obtenir une description des comportements moraux dont il veut ensuite donner la conceptualisation en dehors de toute forme de réification. La mise en concept, si elle veut rester relativiste au sens que Simmel accorde à ce terme, doit chaque fois mettre en évidence la non fermeture de la perspective sur tel aspect de la vie. C'est l'avantage de la Wechselwirkung que d'offrir à la réflexion cet infini, et pour Simmel, cet infini signifie la Vie avec un grand V. La Vie, c'est le moment métaphysique par excellence de la théorie de Simmel. Mais ce n'est pas une métaphysique au sens scolastique du mot que Simmel désire développer, ni complètement un doublage du vitalisme bergsonien ${ }^{57}$. C'est le tragique de la Vie qui intéresse avant tout

57 Certes, Simmel s'est bien laissé influencé par Bergson, mais surtout en ce qui concerne la conception du temps. Simmel montre que le concept de temps en tant que tel est vide, et que le temps se réduit à la temporalité vécue, comme il en va de façon analogue chez Mead. Ceci permet à Simmel d'aboutir à la réflexion sur la vie comme plus-vie et plus-que-vie. Le présent n'est que la collision du passé et du futur; il est donc le moment entre ce qui n'est plus et ce qui n'est pas encore. Ou 
Simmel, et qu'il présente dans ses commentaires de Schopenhauer et Nietzsche par exemple. Cette Vie, en tant qu'elle se fonde sur les Wechselwirkungen, devient tragique par le fait qu'elle ne se dessine toujours que dans le mouvement entre opposés, et donc dans l'impossibilité de la conciliation ${ }^{58}$.

Nous n'avons qu'évoqué ce point dans notre article, et nous ne le développerons pas plus avant. Il nous a seulement permis de comprendre que même lorsque Simmel développe une forme spéciale de métaphysique, il en rappelle le fondement relationniste, qui bien qu'absolutisé n'en constitue pour lui pas moins l'objet propre des sciences humaines. C'est, a posteriori, reconnaître toute l'importance des éléments amenés lors de l'élaboration de la dimension sociologique de la Wechselwirkung. C'est là que Simmel propose de s'attacher à la compréhension des relations sociales et en général des processus sociaux. Il fonde sur cette base une démarche menant à la découverte des façons dont la société est possible, à partir des trois principaux niveaux selon lesquelles elle se concrétise. Le relationnel pris comme objet privilégié de la sociologie suscite la recherche d'une méthode propice à l'investigation de sa variété, la 'sociologie pure' (G. Simmel, (1917)). L'appellerions-nous aujourd'hui une sociologie de la troisième voie? Nous aimerions avoir donné au lecteur quelques éléments pour qu'il puisse creuser plus loin que nous et autrement cette dernière suggestion.

Faculté de Sociologie

Université de Bielefeld

\section{BIBLIOGRAPHIE}

C. Adair-Toteff, «Kant's Philosophical Influence on Classical German Sociology: Simmel's 'Exkursus über das Problem: Wie ist Gesellschaft möglich?'», Simmel Newsletter, Bielefeld, Georg Simmel-Gesellschaft (eds), vol. 4, n²1, 1994, pp. 3-9.

Ch. Andler, V. Basch, J. Benrubi, C. Bouglé, V. Delbos, G. Dwelshauvers, B. Groethuysen, H. Norero, La philosophie allemande au XIXè siècle, Paris, Alcan, 1912.

R. Aron, La sociologie allemande contemporaine, Paris, Quadrige, (1935) 1981.

H. J. Becher, Georg Simmel. Die Grundlagen seiner Soziologie, Stuttgart, Ferdinand Enke, 1971.

R. Boudon, Etudes sur les sociologues classiques, Paris, Quadrige/PUF, 1998.

C. Bouglé, Les sciences sociales en Allemagne. Le conflit des méthodes, Paris, Alcan, (1896) 1912.

B. Chokran, Les pauvres, Paris, Quadrige/PUF, 1998.

plus exactement, il se constitue à travers ces deux moments. C'est ainsi que la vie se révèle comme existence et acquière sa réalité. Le présent à donc une durée, ce que dit également Mead. La condition de cette durée est, pour Simmel comme pour Bergson et Kant, la mémoire. Pour des développements analogues, nous renvoyons le lecteur à Kintzlé (J. Kintzlé, 1988), à l'introduction de Landmann dans Das indivduelle Gesetz. Philosophische Exkurse ((1968) 1987) et à G. Fitzi (1999).

58 Nous suivons en ce sens le propos de Landmann: «Man könnte dieses Prinzip abkürzend als 'Dialektik ohne Versöhnung' bezeichnen.» (M. Landmann, (1968) 1987, p. 16). Simmel ne pense pas ici à une unité métaphysique, mais à un univers pluriel ('pluralistischen Universum'; M. Landmann, (1968) 1987, pp. 16-17). L'auteur affirme même: «Die Kategorien schon des frühen Simmel der 'Relativität' im Erkenntnisbereich, der 'Wechselwirkung' im sozialen haben hier ihren Ursprung.» (M. Landmann, (1968) 1987, p. 17). 
P. Christian, Einheit und Zwiespalt. Zum hegelianisierenden Denken in der Philosophie und Soziologie Georg Simmels, Berlin, Duncker und Humbolt, 1978.

L. A. Coser, Masters of Sociological Thought: Ideas in Historical and Social Context, New York, Harcourt Brace Jovanovich, 1977.

H.-J. Dahme, O. Rammstedt, Georg Simmel und die Moderne, Frankfurt am Main, Suhrkamp, 1984.

L. Deroche-Gurcel, S. Muller, Sociologie : Étude sur les formes de la socialisation, Paris, PUF, 1999.

W. Dilthey, Der Aufbau der Geschichtlichen Welt in den Geisteswissenschaften, Stuttgart, Vandenhoeck et Ruprecht, vol. VII., (1883) 1961, ch. 4.

E. Durkheim, Eléments d' une théorie sociale, Paris, Minuit, V. Karady (eds), vol. I, 1975.

J. G. Fichte, «Aenesidemus oder über die Fundamente von dem Herrn Professor Reinhold in Jena gelieferten Elementar-Philosophie. Nebst einer Vertheidigung des Skepticismus gegen die Anmaassungen der Vernunftkritik», Johann Gottlieb Fichtes sämmtliche Werke, Berlin, Veit \& Comp., I. H. Fichte (eds), vol. I, (1792) 1845-1846: 4-25.

- «Grundriss des Eigenthümlichen der Wissenschaftslehre», Johann Gottlieb Fichtes sämmtliche Werke, Berlin, Veit \& Comp., I. H. Fichte (eds), vol. I (1795) 1845-1846, pp. 329-411.

- «Grundlage des Naturrechts », Johann Gottlieb Fichtes sämmtliche Werke, Berlin, Veit \& Comp., I. H. Fichte (eds), vol. III, (1796) 1845-1846, pp. XLVIII-385.

G. Fitzi, Henri Bergson und Georg Simmel : ein Dialog zwischen Leben und Krieg. Die persönliche Beziehung und der wissenschaftliche Austausch zweier Intellektuellen im deutsch-französischen Kontext vor dem Ersten Weltkrieg, Bielefeld, Faculté de Sociologie, thèse de doctorat, 1999.

A. Frank, «Review Essay», Symbolic Interaction, n 10 (2), 1997: 295-311.

J. Freund, «Introduction», Georg Simmel. Sociologie et épistémologie, Paris, PUF, (1981) 1991, pp. $7-83$.

- «Note critique sur 'La philosophie de l'argent' de Georg Simmel», L'Année Sociologique, n`39, 1989, pp. 272-297.

- «De Max Weber à Georg Simmel », Sociétés, n³7, Paris, Dunod, 1991, pp. 217-224.

- «Philosophie des Geldes. La référence méthodologique de l'interprétation de la pensée de G. Simmel.», G. Simmel et les sciences humaines. Actes du Colloque G. Simmel et les sciences humaines 14-15 septembre 1988, Paris, Méridiens Klincksieck, O. Rammstedt et P. Watier (eds), 1992, pp. 63-77.

D. Frisby, Sociological Impressionism : A Reassessment of Georg Simmel's Social Theory, London, Heinemann, 1981.

- Georg Simmel, New-York, Ellis Horwood, 1984.

J. W. von Goethe, «Es gibt keine patriotische Kunst», Goethes Werke, München, Deutscher Taschenbuch Verlag, P. Raabe (eds), vol. 42, T. I, [(1832) 1919] 1990, pp. 195-196.

- Die Leiden des jungen Werthers, Stuttgart, Reclam, 1995.

A. Hahn, «Mort et civilisation chez Simmel», Actes du colloque Georg Simmel-Gesellschaft, Strasbourg, Bielefeld, 1988, pp. 1-9.

G. W. F. Hegel, Enzyklopädie der philosophischen Wissenschaften, Berlin, Hegel-Institut Berlin (eds), vol. I, (1830) 1998.

- Wissenschaft der Logik, Berlin, Hegel-Institut Berlin (eds), vol. II, (1831) 1998.

V. Jankelevitch, «Georg Simmel, philosophie de la vie», Revue de Métaphysique et de Morale, Paris, Armand Colin, vol. XXXII, 1925, pp. 213-257 et 373-386.

I. Kant, «Allgemeine Naturgeschichte und Theorie des Himmels», Kants gesammelten Schriften, Leipzig, Dürr, Preussischen Akademie der Wissenschaften (eds), vol. I, (1755) 1902-1923, pp. 215-366.

- « Nachricht von der Einrichtung seiner Vorlesungen in dem Winterhalbenjahre von 1765-1766», Kants gesammelten Schriften, Leipzig, Dürr, Preussischen Akademie der Wissenschaften (eds), vol. II, (1765-66) 1902-1923, pp. 305-313.

- $\quad$ «Täume eines Geistersehers » Kants gesammelten Schriften, Leipzig, Dürr, Preussischen Akademie der Wissenschaften (eds), vol. II, (1766) 1902-1923, pp. 315-373. 
- Kritik der reinen Vernunft, Frankfurt am Main, Suhrkamp, W. Weischedel (eds), (1781/1787) 1995.

J. Kintzelé, «Sociabilité et temporalité chez Simmel: Vers une sociologie du présent », Actes du colloque Georg Simmel-Gesellschaft, Strasbourg, Bielefeld, 1988, pp. 1-13.

M. Landmann, Introduction, « Georg Simmel: Konturen seines Denkens », Ästhetik und Soziologie um die Jahrhundertwende: Georg Simmel, Frankfurt am Main, H. Böhringer, K. Gründer (eds), 1976: 3-17.

- « Einleitung des Herausgebers », Das individuelle Gesetz. Philosophische Exkurse, Frankfurt am Main, Suhrkamp, M. Landmann (eds), (1968) 1987, pp. 7-30.

D. N. Levine, «Simmel as a Ressource for Sociological Metatheory », Actes du colloque Georg Simmel-Gesellschaft, Strasbourg, Bielefeld, 1988.

K. Lichtblau, Georg Simmel, Frankfurt, New York, Reihe Campus, 1997.

I. Newton, Optique, Paris, Bourgeois, (1787) 1989.

R. A. Nisbet, «The Molecular Community - Simmel», The Sociological Tradition, New York, Basic Books, 1966: 96-106.

C. Papilloud, «Simmel, Durkheim et Mauss. La sociologie entre l'Allemagne et la France. Fragments d'une co-naissance », Simmel Newsletter, Bielefeld, Georg Simmel Gesellschaft (eds), vol. 9, n² , 1999, pp. 114-132.

W. Pfeifer, «Wechsel» et «Wirken», Etymologischer Wörterbuch des Deutschen, Berlin, AkademieVerlag Berlin, 1989.

O. Rammstedt, «La 'Soziologie’ de 1908 de Georg Simmel», Sociétés, Paris, Dunod, n`37, 1991, pp. 253-266.

- «Georg Simmel et la fondation de la sociologie», G. Simmel et les sciences humaines. Actes du Colloque G. Simmel et les sciences humaines 14-15 septembre 1988, Paris, Méridiens Klincksieck, O. Rammstedt et P. Watier (eds), 1992, pp. 107-131.

F. D. E. Schleiermacher, Enfwürfe zu einem System der Sittenlehre, Leipzig, O. Braun (eds), Felix Meiner, Vol. 2, (1927), pp. 1-33.

A. W. von Schlegel, Über Literatur, Kunst und Geist des Zeitalters : eine Auswahl aus den kritischen Schriften, Stuttgart, F. Finke (eds), Reclam, (1802) 1994.

O. Shade, «Wechsel» et «Wirken», Altdeutsches Wörterbuch, Königsberg, Halle, A. Ds, vol. 2, 1882.

G. Simmel, Das Wesen der Materie nach Kant's Physischer Monadologie, Berlin, Druck der Norddeutschen Buchdruckerei, 1881.

G. Simmel, «Panta rhei », Simplizissimus, Kriegsflugblaetter, München, n 6, 1917, p. 274.

G. Simmel, «Aus Georg Simmels nachgelassenem Tagebuch», Logos, n8, 1919/1920, pp. 121-151.

G. Simmel, Grundfragen der Soziologie, Berlin, W. de Gruyter \& Co., 1984.

G. Simmel, Das Wesen der Materie (1881). Abhandlungen 1882-1884. Rezensionen 1883-1901, GSG 1, Frankfurt am Main, K. Ch. Köhnke, O. Rammstedt, Suhrkamp, 2000.

- Aufsätze 1887-1890. Über sociale Differenzierung (1890). Die Probleme der Geschichtsphilosophie (1892), GSG 2, Frankfurt am Main, H.-J. Dahme, O. Rammstedt (eds), Suhrkamp, 1989.

- Einleitung in die Moralwissenschaft, Band I (1892/1904), GSG 3, Frankfurt am Main, K. Ch. Köhnke, O. Rammstedt (eds), Suhrkamp, 1989.

- Einleitung in die Moralwissenschaft, Band II (1893), GSG 4, Frankfurt am Main, K. Ch. Köhnke, O. Rammstedt (eds), Suhrkamp, 1989.

- Aufsätze und Abhandlungen 1894-1900, GSG 5, Frankfurt am Main, H.-J. Dahme, D. P. Frisby, O. Rammstedt (eds), Suhrkamp, 1992.

- Philosophie des Geldes (1900/1907), GSG 6, Frankfurt am Main, David P. Frisby, K. Ch. Köhnke, O. Rammstedt (eds), Suhrkamp, 1996.

- Aufsätze und Abhandlungen 1901-1908, Band I, GSG 7, Frankfurt am Main, R. Kramme, A. Rammstedt, O. Rammstedt (eds), Suhrkamp, 1995. 
- Aufsätze und Abhandlungen 1901-1908, Band II, GSG 8, Frankfurt am Main, A. Cavalli, V. Krech, O. Rammstedt (eds), Suhrkamp, 1993.

- Philosophie der Mode (1905). Die Religion (1906/1912). Kant und Goethe (1906/1916). Schopenhauer und Nietzsche (1907), GSG 10, Frankfurt am Main, M. Behr, V. Krech, G. Schmidt, O. Rammstedt (eds), Suhrkamp, 1998.

- Soziologie (1908), GSG 11, Frankfurt am Main, K. Ch. Köhnke, O. Rammstedt (eds), Suhrkamp, 1992.

- Hauptprobleme der Philosophie. Philosophische Kultur, GSG 14, Frankfurt am Main, R. Kramme, O. Rammstedt (eds), Suhrkamp, 1996.

- Grundfragen der Soziologie (1917). Der Krieg und die geistigen Entscheidungen (1917). Der Konflikt der modernen Kultur (1918). Vom Wesen des historischen Verstehens (1918). Lebensanschauung (1918), GSG 16, Frankfurt am Main, G. Fitzi, O. Rammstedt (eds), Suhrkamp, 1998.

N. J. Spykman, The Social Theory of Georg Simmel, Ipswich, Suffolk, Ipswich Book Co, (1925) 1992.

G. Tarde, Psychologie Economique, Paris, Alcan, T. I, 1902.

F. Vandenberghe, «La double dualité comme principe d'unité de la pensée simmelienne», Simmel Newsletter, Bielefeld, Georg Simmel-Gesellschaft (eds), vol. 9, n² 2, 1999, pp. 132-144.

- Sociologie de Georg Simmel, Paris, La Découverte, Repères, (à paraître).

D. Weinstein, M. A. Weinstein, Postmodern(ized) Simmel, London, New York, Routledge, 1993. 